\title{
Broadband Performance Metrics and Regression Approximations of the New Coupling Schemes for Distribution Broadband over Power Lines (BPL) Networks
}

\author{
Athanasios G. Lazaropoulos ${ }^{*}$ \\ School of Electrical and Computer Engineering / National Technical University of Athens / \\ 9 Iroon Polytechniou Street / Zografou, GR 15780
}

Received February 05, 2018; Accepted March 11, 2018; Published March 15, 2018

\begin{abstract}
This paper assesses the broadband performance of overhead (OV) and underground (UN) low-voltage (LV) and medium-voltage (MV) broadband over power lines (BPL) networks when the new refined Coupling Scheme module (CS2 module) is adopted. The broadband performance of distribution BPL networks is assessed in terms of their Average Channel Gain (ACG), Root-Mean-Square Delay-Spread (RMS-DS), Coherence Bandwidth (CB) and Spectral Efficiency (SE). Also, corresponding regression approximations (i.e., UN1, UN2 and UN3 approaches) are given in the examined BPL frequency range. The aforementioned broadband performance metrics of the application of CS2 module are compared against the relative ones of the vintage CS1 module and of MIMO channels. The analysis and relevant numerical results outline: (i) the important improvement of the aforementioned performance metrics and regression approximations when CS2 module is applied in distribution BPL networks instead of CS1 module; and (ii) the universal role of UN1, UN2 and UN3 approaches for describing coupling scheme channels and MIMO ones.
\end{abstract}

Keywords: Broadband over Power Lines (BPL); Power Line Communications (PLC); distribution power grids; statistical performance metrics; Smart Grid (SG).

\section{Introduction}

The evolution of the traditional distribution power grids -i.e., overhead $(\mathrm{OV})$ and underground (UN) low-voltage (LV) and medium-voltage (MV)- to a unified smart grid can become the key to delivering broadband last-mile access and simultaneously to developing of an advanced IP-based power system [1]-[7]. Actually, the smart grid is a systems epitome that embodies the interoperability of several communications technologies [8], [9]. Among the candidate communications solutions, broadband over power lines networks (BPL) networks attract the decision-makers' interest due to their techno-economic advantage of not requiring further investments concerning their network cabling since the infrastructure of distribution power grids acts as the required network communication backbone [10]. 
However, the propagation and transmission of communications signals along distribution BPL networks remain a challenging issue since distribution power grids were not designed for broadband communications purposes. This rather hostile medium for communications is characterized by high attenuation, multipath due to various reflections, BPL signal coupling losses, noise and electromagnetic interference (EMI) [11]-[16]. It is evident that each of the aforementioned aggravating factors differently influences the performance of distribution BPL networks.

As the attenuation, multipath due to various reflections and coupling losses are concerned, the well-validated hybrid model achieves to accurately describe them in BPL networks [2]-[4], [6], [7], [11], [14], [17]-[25]. Being extensively verified during the performance assessment of various multiconductor transmission line (MTL) configurations in BPL networks, the hybrid model consists of two interconnected methods, namely: (i) a bottom-up method that is based on an appropriate combination of MTL theory and similarity transformations, such as the EigenValue Decomposition (EVD), the Single Value Decomposition (SVD) and the Unified Value Decomposition (UVD); and (ii) a top-down method that is based on the concatenation of multidimensional transmission matrices of the cascaded network BPL connections. Various refinements of the aforementioned two methods have been proposed in the literature in order to cope with the special needs of the various supported smart grid applications (e.g., Multiple Input Multiple Output (MIMO) BPL networks [25], [26], power systems stability [27]-[29], identification and localization of faults across distribution power grids [30] and wireless sensor networks [31], [32]).

However, one recent refinement of the top-down method that can significantly enhance the broadband performance and influence the future architectural design of BPL networks is the proposal of Coupling Scheme module (CS2) module [33]. Similarly to its predecessor CS1 module [3], [6], CS2 method handles with the injection and the extraction of BPL signals across the power lines of distribution power grids. As already been presented in [33], findings concerning channel attenuation and capacity reveal that CS2 module better exploits all the available conductors of the MTL configurations than its predecessor CS1 module through the wiser management of the BPL signal power injection and extraction.

In this paper, the broadband performance metrics of Average Channel Gain (ACG), Root-Mean-Square Delay-Spread (RMS-DS), Coherence Bandwidth (CB) and Spectral Efficiency (SE) of OV and UN MV and LV BPL networks when CS2 module is applied are compared against the respective ones of CS1 module and MIMO channels [17]. Besides the comparison of performance metrics and the relative observations, the regression approximations of [17], i.e., UN1 and $\mathrm{UN} 2$ approaches, are again calculated for OV and UN LV and MV BPL networks by taking into account the configuration of CS2 module. In addition, UN3 approach that relates SE with ACG is proposed for OV and UN LV and MV BPL networks. Finally, the detailed UN1 and UN2 approaches of CS2 module are compared against the respective ones of CS1 module and of MIMO channels while UN3 approach is assessed for CS2 and CS1 module. The universal character of these three approaches in describing distribution BPL networks is here validated.

The rest of this paper is organized as follows: In Section II, OV and UN LV and MV configurations used in this paper are briefly presented. Section III synopsizes the basics of the propagation, the transmission and the signal coupling across distribution BPL networks. Here, CS2 module is detailed while its operation and performance are 
compared against the ones of CS1 module. Section IV reports the broadband performance statistical metrics as well as UN1, UN2 and UN3 regression approximations, which are applied in this paper. In Section V, a series of numerical results and conclusions are provided, aiming at marking out the impact of various coupling schemes supported by CS2 module on the statistical performance metrics and regression approximations of distribution BPL networks. Section VI concludes this paper.

\section{Distribution BPL Network Configurations}

\subsection{MTL Configurations of OV and UN LV and MV BPL Networks}

In Figs. 1(a)-(d) of [6], the MTL configurations of distribution BPL networks that are examined in this paper are demonstrated. More specifically:

- OV LV MTL Configuration: With reference to Fig. 1(a) of [6], four parallel non-insulated conductors are suspended one above the other spaced by $\Delta_{L V}$ while the lowest conductor is suspended at height $h_{L V}$ above ground $\left(n^{\text {OVLV }}=4\right)$. The upper conductor is the neutral, while the lower three conductors are the three phases. The exact dimensions for this MTL configuration are detailed in [6].

- OV MV MTL Configuration: With reference to Fig. 1(b) of [6], the horizontal arrangement of OV MV distribution lines consists of three parallel non-insulated phase conductors $\left(n^{\text {OVMV }}=3\right)$ spaced by $\Delta_{M V}$ that are hang at typical height $h_{M V}$ above ground. There is no neutral conductor. The exact properties of this MTL configuration are given in [6].

- UN LV MTL Configuration: With reference to Fig. 1(c) of [6], the cable arrangement of UN LV MTL configuration consists of the three-phase three-core-type conductors, one core-type neutral conductor and one shield conductor $\left(n^{\mathrm{UNLV}}=4\right)$. The shield is grounded at both ends while the cable is buried $1 \mathrm{~m}$ inside the ground. The exact dimensions of this UN LV MTL configuration are reported in [6].

- UN MV MTL Configuration: With reference to Fig. 1(d) of [6], the cable arrangement of UN MV MTL configuration comprises the three-phase three- sector-type conductors, one shield conductor and one armor conductor. Similarly to UN LV MTL configuration, both the shield and the armor are grounded at both ends while the cable is buried $1 \mathrm{~m}$ inside the ground. Due to the shielding of UN MV cables and according to [2], [6], [34], the propagation and transmission analysis can be only focused on the inner set of conductors ( $n^{\mathrm{UNMV}}=3$ ), say the three-phase conductors and the shield. The exact dimensions of this UN MV MTL configuration are given in [6].

Finally, the properties of the ground, which are detailed in [6], are assumed common in distribution BPL networks. Here, it should be noted that these ground properties are suitable for the propagation and transmission of BPL signal across distribution BPL networks that operate in the $1-100 \mathrm{MHz}$ frequency range of interest.

\subsection{Indicative Distribution BPL Topologies}

To define a set of indicative distribution BPL topologies, the simple BPL topology of Fig. 1 is considered. With reference to Fig. 1, the transmitting and receiving ends, which are situated at point A and B of the simple BPL topology, respectively, 
are assumed to be matched. The branch terminations, which are connected at positions $A_{k}^{\prime}, k=1, \ldots N$, are assumed open circuits [11], [13]-[16], [35]-[38]. The end-to-end connection between transmitting and receiving end (path length) of the simple BPL topology, which is encountered in BPL signal transmission, is equal to $L=\sum_{k=1}^{N+1} L_{k}$ $[38]-[40]$.

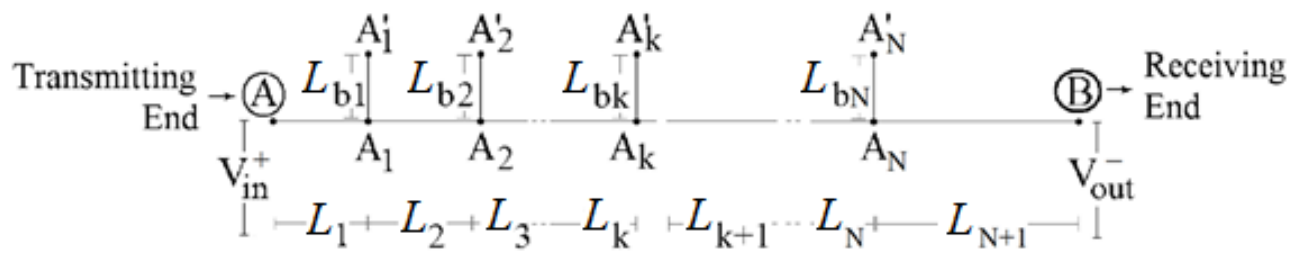

Fig. 1. Simple BPL topology [14], [16].

For comparison reasons, same indicative distribution BPL topologies with the ones of [6], [33] are considered in this paper. In Table 1, five indicative OV distribution BPL topologies of $1000 \mathrm{~m}$ average path lengths are given as well as the correspondent description, the number of branches, the length of distribution lines and the length of branch lines. Similarly to Table 1, five indicative UN distribution BPL topologies of $200 \mathrm{~m}$ average path length with their properties are reported in Table 2. Here it should be noted that for given type of distribution power grids, the topologies remain the same; say, BPL topologies of Table 1 remain the same either OV LV or OV MV power grid is considered.

Table 1

OV Distribution BPL Topologies

\begin{tabular}{|c|c|c|c|c|}
\hline $\begin{array}{c}\text { Topology } \\
\text { Name }\end{array}$ & Topology Description & $\begin{array}{c}\text { Number of } \\
\text { Branches }\end{array}$ & $\begin{array}{c}\text { Length of } \\
\text { Distribution Lines }\end{array}$ & $\begin{array}{c}\text { Length of Branch } \\
\text { Lines }\end{array}$ \\
\hline Urban case A & $\begin{array}{c}\text { Typical overhead urban } \\
\text { topology }\end{array}$ & 3 & $\begin{array}{c}\mathrm{L}_{1}=500 \mathrm{~m}, \\
\mathrm{~L}_{2}=200 \mathrm{~m}, \\
\mathrm{~L}_{3}=100 \mathrm{~m}, \mathrm{~L}_{4}=200 \mathrm{~m}\end{array}$ & $\begin{array}{c}\mathrm{L}_{\mathrm{b} 1}=8 \mathrm{~m}_{,} \mathrm{L}_{\mathrm{b} 2}=13 \mathrm{~m}, \\
\mathrm{~L}_{\mathrm{b} 3}=10 \mathrm{~m}\end{array}$ \\
\hline Urban case B & $\begin{array}{c}\text { Aggravated overhead urban } \\
\text { topology }\end{array}$ & 5 & $\begin{array}{c}\mathrm{L}_{1}=200 \mathrm{~m}, \mathrm{~L}_{2}=50 \mathrm{~m}, \\
\mathrm{~L}_{3}=100 \mathrm{~m}, \\
\mathrm{~L}_{4}=200 \mathrm{~m}, \\
\mathrm{~L}_{5}=300 \mathrm{~m}, \mathrm{~L}_{6}=150 \mathrm{~m}\end{array}$ & \begin{tabular}{c}
$\mathrm{L}_{\mathrm{b} 1}=12 \mathrm{~m}, \mathrm{~L}_{\mathrm{b} 2}=5 \mathrm{~m}$, \\
$\mathrm{L}_{\mathrm{b} 3}=28 \mathrm{~m}$, \\
$\mathrm{L}_{\mathrm{b} 4}=41 \mathrm{~m}, \mathrm{~L}_{\mathrm{b} 5}=17 \mathrm{~m}$ \\
\hline Suburban case
\end{tabular} \\
$\begin{array}{c}\text { Overhead suburban topology } \\
\mathrm{L}_{1}=500 \mathrm{~m},\end{array}$ & 2 & $\mathrm{~L}_{\mathrm{b} 1}=50 \mathrm{~m}, \mathrm{~L}_{\mathrm{b} 2}=10 \mathrm{~m}$ \\
\hline Rural case & Overhead rural topology & 1 & $\mathrm{~L}_{1}=600 \mathrm{~m}, \mathrm{~L}_{2}=400 \mathrm{~m}$ & $\mathrm{~L}_{\mathrm{b} 1}=300 \mathrm{~m}$ \\
\hline "LOS" case & $\begin{array}{c}\text { Overhead Line-of-Sight } \\
\text { transmission }\end{array}$ & 0 & $\mathrm{~L}_{1}=1000 \mathrm{~m}$ & - \\
\hline
\end{tabular}


Table 2

UN Distribution BPL Topologies

\begin{tabular}{|c|c|c|c|c|}
\hline $\begin{array}{c}\text { Topology } \\
\text { Name }\end{array}$ & Topology Description & $\begin{array}{c}\text { Number of } \\
\text { Branches }\end{array}$ & $\begin{array}{c}\text { Length of } \\
\text { Distribution Lines }\end{array}$ & $\begin{array}{c}\text { Length of Branch } \\
\text { Lines }\end{array}$ \\
\hline Urban case A & $\begin{array}{c}\text { Typical underground urban } \\
\text { topology }\end{array}$ & 3 & $\begin{array}{c}\mathrm{L}_{1}=70 \mathrm{~m}, \mathrm{~L}_{2}=55 \mathrm{~m}, \\
\mathrm{~L}_{3}=45 \mathrm{~m}, \mathrm{~L}_{4}=30 \mathrm{~m}\end{array}$ & $\begin{array}{c}\mathrm{L}_{\mathrm{b} 1}=12 \mathrm{~m}, \\
\mathrm{~L}_{\mathrm{b} 2}=7 \mathrm{~m}, \\
\mathrm{~L}_{\mathrm{b} 3}=21 \mathrm{~m}\end{array}$ \\
\hline Urban case B & $\begin{array}{c}\text { Aggravated underground } \\
\text { urban topology }\end{array}$ & 5 & $\begin{array}{c}\mathrm{L}_{1}=40 \mathrm{~m}, \mathrm{~L}_{2}=10 \mathrm{~m}, \\
\mathrm{~L}_{3}=20 \mathrm{~m}, \mathrm{~L}_{4}=40 \mathrm{~m}, \\
\mathrm{~L}_{5}=60 \mathrm{~m}, \mathrm{~L}_{6}=30 \mathrm{~m}\end{array}$ & $\begin{array}{c}\mathrm{L}_{\mathrm{b} 1}=22 \mathrm{~m}, \\
\mathrm{~L}_{\mathrm{b} 4}=12 \mathrm{~m}, \mathrm{~L}_{\mathrm{b} 3}=8 \mathrm{~m}, \mathrm{~L}_{\mathrm{b} 5}=17 \mathrm{~m}\end{array}$ \\
\hline Suburban case & $\begin{array}{c}\text { Underground suburban } \\
\text { topology }\end{array}$ & 2 & $\mathrm{~L}_{1}=50 \mathrm{~m}, \mathrm{~L}_{2}=100 \mathrm{~m}$, \\
$\mathrm{L}_{3}=50 \mathrm{~m}$ & $\mathrm{~L}_{\mathrm{b} 1}=60 \mathrm{~m}, \mathrm{~L}_{\mathrm{b} 2}=30 \mathrm{~m}$ \\
\hline Rural case & Underground rural topology & 1 & $\mathrm{~L}_{1}=50 \mathrm{~m}, \mathrm{~L}_{2}=150 \mathrm{~m}$ & $\mathrm{~L}_{\mathrm{b} 1}=100 \mathrm{~m}$ \\
\hline "LOS" case & $\begin{array}{c}\text { Underground Line-of-Sight } \\
\text { transmission }\end{array}$ & 0 & $\mathrm{~L}_{1}=200 \mathrm{~m}$ & - \\
\hline
\end{tabular}

\section{Relationship among BPL Signal Coupling, Transmission and Propagation Distribution BPL Networks}

With reference to Fig. 1, the input BPL signal $V_{\text {in }}^{+}$, which is measured at the transmitting end, carries all the required information that needs to be transmitted through the BPL topology while the output BPL signal $V_{\text {out }}^{-}$, which is measured at the receiving end, should optimally (i.e., if aggravating factors are ignored) be equal to the input BPL signal so that no information is lost. However, aggravating factors that include the attenuation due to the BPL signal propagation, multipath due to the BPL signal transmission and coupling losses influence the measured output BPL signal.

In Figs. 2(a) and 2(b), the general implementation of a coupling scheme module that describes the injection of the input BPL signal onto and the extraction of the output BPL signal from the power lines of BPL topologies, respectively, is illustrated. In fact, different power percentages are allocated among the available conductors that depend on: (i) the participation percentages $C_{i}^{\text {in }}, i=1, \ldots, n^{\mathrm{G}}$ of the conductors of the MTL configuration during the BPL signal injection; and (ii) the participation percentages $C_{i}^{\text {out }}, i=1, \ldots, n^{\mathrm{G}}$ of the conductors of the MTL configuration during the BPL signal

extraction. Note that $C_{i}^{\mathrm{in}}, i=1, \ldots, n^{\mathrm{G}}$ and $C_{i}^{\text {out }}, i=1, \ldots, n^{\mathrm{G}}$ are the elements of the $n^{\mathrm{G}} \times 1$ input coupling column vector $\mathrm{C}^{\mathrm{in}}$ and the $1 \times n^{\mathrm{G}}$ output coupling line vector $\mathrm{C}^{\text {out }}$, respectively, where []$^{\mathrm{G}}$ denotes the examined power grid type -either OV MV or UN MV or OV LV or UN LV-. 


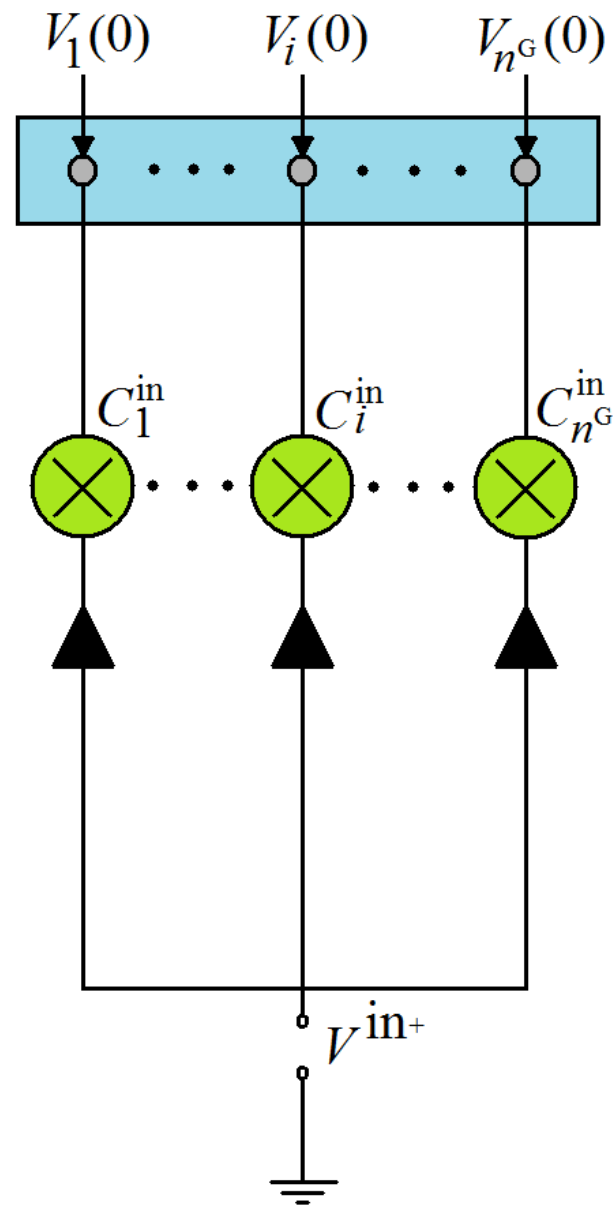

(a)

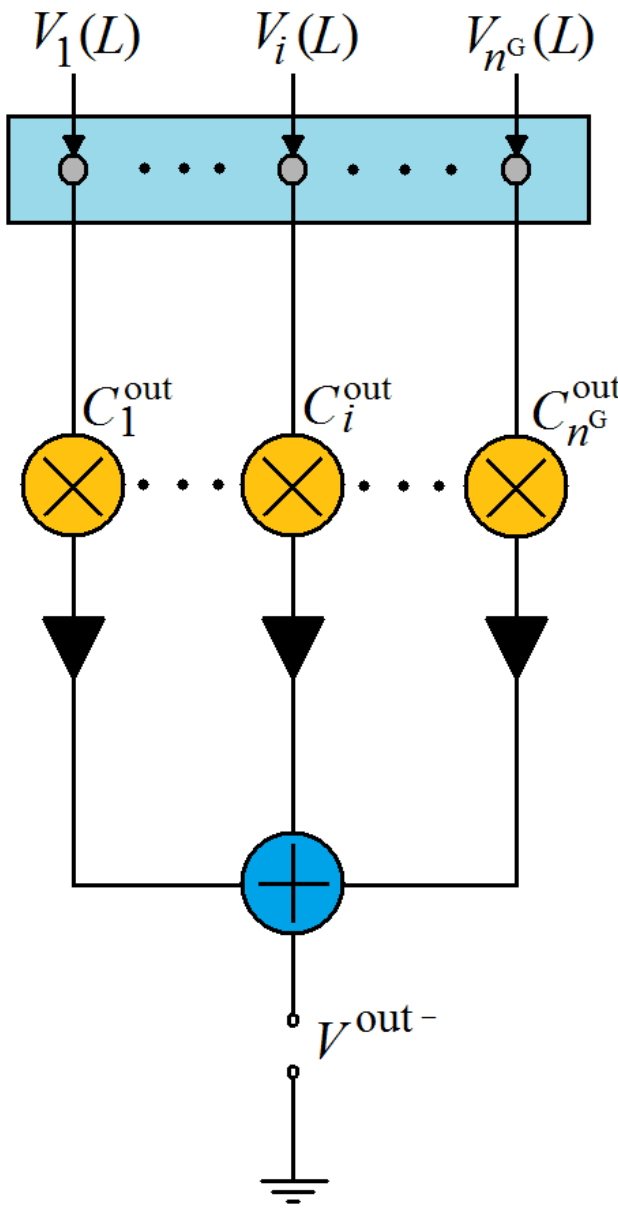

(b)

Fig. 2. General implementation of a coupling scheme module [33]. (a) BPL signal injection interface at the transmitting end. (b) BPL signal extraction at the receiving end.

Depending on the element values of $\mathbf{C}^{\text {in }}$ and $\mathbf{C}^{\text {out }}$ that are restricted by a set of power related constraints analyzed in [33], coupling scheme modules can support three different types of coupling schemes, namely:

1. Coupling Scheme Type 1: It consists of Wire-to-Ground (WtG) or Shield-to-Phase (StP) coupling schemes for OV or UN distribution BPL networks, respectively. The signal injection is made in only one conductor at the transmitting end and returns via either the ground for OV distribution cables or the shield for UN distribution cables. The signal extraction is made from the same conductor at the receiving end. $\left[\mathbf{C}^{\text {in }}\right]^{\mathrm{WtG}^{\mathrm{S}} / \mathrm{StP}^{\mathrm{s}}}$ and $\left[\mathbf{C}^{\text {out }}\right]^{\mathrm{Wt \textrm {G } ^ { \mathrm { s } }} / \mathrm{StP}^{\mathrm{s}}}$ have zero elements except in line $\mathrm{s}$ and row $\mathrm{s}$, respectively, where the value is equal to 1 . Note that $[\cdot]^{\mathrm{C}}$ denotes the applied coupling scheme. Both CS1 and CS2 modules support this coupling scheme type without performance diversification.

2. Coupling Scheme Type 2: It consists of Wire-to-Wire (WtW) or Phase-to-Phase (PtP) coupling schemes for OV or UN distribution BPL networks, respectively. The signal is injected in equal parts between two conductors while it 
is extracted from the same conductors. $\left[\mathbf{C}^{\text {in }}\right]^{\mathrm{WtW} \mathrm{W}^{p-q} / \mathrm{PtP}^{p-q}}$ and $\left[\mathbf{C}^{\text {out }}\right]^{\mathrm{WtW}^{p-q} / \mathrm{PtP}^{p-q}}$ have zero elements except in lines $p$ and $q$ where the values are different from zero. In terms of these non-zero values, CS1 and CS2 module differentiate each other (see the following analysis).

3. Coupling Scheme Type 3: It consists of MultiWire-to-MultiWire (MtM) or MultiPhase-to-MultiPhase (MtM) coupling schemes for OV or UN distribution BPL networks, respectively. The signal is injected among multiple conductors with different participation percentages while the signal is extracted from the same conductor set at the receiving end. For example, when MtM coupling scheme occurs among the three conductors $p, q$ and $r, p, q, r=1, \ldots, n^{\mathrm{G}}$ of $\mathrm{MV}$ BPL networks with participation percentages equal to $C_{p}^{\text {in }}, C_{q}^{\text {in }}$ and $C_{r}^{\text {in }}$, respectively, $\left[\mathbf{C}^{\text {in }}\right]^{\mathrm{MtM}_{C_{\mathrm{p}}-c_{\mathrm{q}}-c_{\mathrm{r}}^{p-q}}^{\text {in }}}$ has zero elements except in lines $p, q$ and $r$.

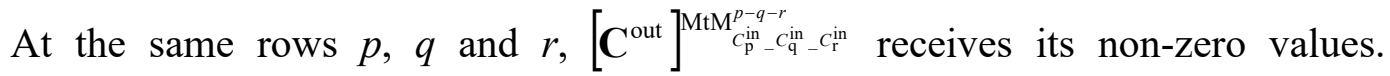
This coupling scheme type is only supported by CS2 module.

In accordance with [33] and the general implementation of a coupling scheme module given in Fig. 2, the coupling scheme channel transfer function $H^{\mathrm{C}}\{\cdot\}$ that relates the output BPL signal with the input BPL signal is determined by:

$$
H^{\mathrm{C}}\{\cdot\}=\frac{\left[V^{\text {out- }}\right]^{\mathrm{C}}}{\left[V^{\text {in+ }}\right]^{\mathrm{C}}}=\left[\mathbf{C}^{\text {out }}\right]^{\mathrm{C}} \cdot \mathbf{H}\{\cdot\} \cdot\left[\mathbf{C}^{\text {in }}\right]^{\mathrm{C}}
$$

where $\mathbf{H}\{\cdot\}$ is the $n^{\mathrm{G}} \times n^{\mathrm{G}}$ channel transfer function matrix that relates line voltages $\mathbf{V}(z)=\left[\begin{array}{lll}V_{1}(z) & \cdots & V_{n^{\mathrm{G}}}(z)\end{array}\right]^{\mathrm{T}}$ at the transmitting $(z=0)$ and the receiving $(z=\mathrm{L})$ ends and is the output of the hybrid model (see [33]). Note that []$^{\mathrm{T}}$ denotes the transpose of a matrix. Therefore, the dependence of the coupling scheme channel transfer function on the applied coupling scheme is expressed by the presence of the input and output coupling vector $\mathbf{C}^{\text {in }}$ and $\mathbf{C}^{\text {out }}$, respectively, when the channel transfer function is given.

As it concerns the characterization of the elements of the channel transfer function matrix $\mathbf{H}\{\cdot\}$ of eq. (1), its elements $H_{i j}\{\cdot\}, i, j=1, \ldots, n$ with $i=j$ are the co-channel (CC) transfer functions, while those with $i \neq j$ are the cross-channel (XC) transfer functions where $H_{i j}, i, j=1, \ldots, n$ denotes the element of matrix $\mathbf{H}\{\cdot\}$ in row $i$ of column $j$. All together, $H_{i j}\{\}, i, j=1, \ldots, n$ are the transfer functions of MIMO channels (either CCs or XCs) whose broadband statistical performance has been examined in [17]. By observing eq. (1), coupling and MIMO channel transfer functions are related through the input and output coupling vector while the broadband performance of coupling channels is investigated and compared against the one of MIMO channels through the broadband performance statistical metrics in this paper.

With reference to the aforementioned coupling scheme types, coupling channels and eq. (1), the differences between CS1 module [3], [6] and CS2 module [33] are focused on the element values of the coupling vectors $\mathbf{C}^{\text {in }}$ and $\mathbf{C}^{\text {out }}$ that, anyway, describe the operation of CS1 and CS2 module, say:

- CS1 module: The main concept of this module is its design and definition simplicity that is preserved through: 


$$
\left[\mathbf{C}^{\text {out }}\right]=\left[\mathbf{C}^{\text {in }}\right]^{\mathrm{T}}
$$

Namely, the configuration of BPL injector and extractor remains the same whereas the power flow changes. Therefore, CS1 module can only support coupling scheme type 1 and coupling type scheme 2 . As concerns the coupling scheme type 1 , the element values of $\left[\mathbf{C}^{\mathrm{in}}\right]^{\mathrm{W} t \mathrm{G}^{\mathrm{s}} / \mathrm{StP}}$ has previously been mentioned. As concerns the coupling scheme type 2, the non-zero values in lines $p$ and $q$ of

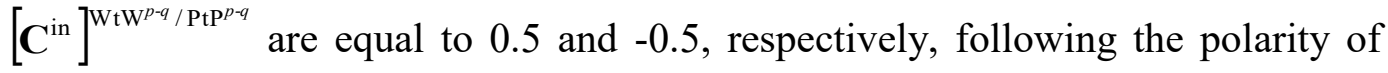
the input signal between the conductors. On the basis of eq. (2), the element values of $\left[\mathbf{C}^{\text {out }}\right]^{\mathrm{WtG}^{s} / \mathrm{StP}^{s}}$ and $\left[\mathbf{C}^{\text {out }}\right]^{\mathrm{Wt} \mathrm{WW}^{p \cdot q} / \mathrm{PtP}^{p \cdot q}}$ can be determined for coupling scheme type 1 and 2, respectively. Note that CS1 module creates coupling losses.

- CS2 module: The main concept of this module is the minimization of coupling losses that is accomplished by the consideration of $\mathbf{C}^{\text {in }}$ and $\mathbf{C}^{\text {out }}$ as orthonormal matrices. Namely, apart from the power flow, the configuration of BPL injector and extractor changes. In fact, the element values of $\mathbf{C}^{\text {in }}$ and $\mathbf{C}^{\text {out }}$ are determined under the principle of energy conservation and eqs (4)-(6) of [33]. With reference to the aforementioned coupling scheme types, CS2 module can support all the three ones. More specifically, as concerns the coupling scheme type 1, CS2 module follows the same definition with CS1 module. As concerns the coupling scheme type 2, CS2 module agrees with the definition of the element values of $\left[\mathbf{C}^{\text {in }}\right]^{\mathrm{WtW}^{p \cdot q} / \mathrm{PPP}^{p \cdot q}}$ but $\left[\mathbf{C}^{\text {out }}\right]^{\mathrm{WtW}} \mathrm{W}^{p \cdot q} / \mathrm{PtP}^{p \cdot q}$ has zero elements except in rows $p$ and $q$ where the values are equal to 1 and -1 , respectively. As concerns the coupling scheme type 3, which is supported only by the CS2 module, when MtM coupling scheme occurs among the three conductors $p, q$ and $r$, $p, q, r=1, \ldots, n^{\mathrm{G}}$ with participation percentages equal to $C_{p}^{\text {in }}, C_{q}^{\text {in }}$ and $C_{r}^{\text {in }}$,

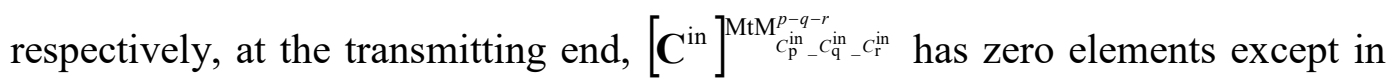
lines $p, q$, and $r$ where the values are equal to $C_{p}^{\text {in }}, C_{q}^{\text {in }}$ and $C_{r}^{\text {in }}$, respectively,

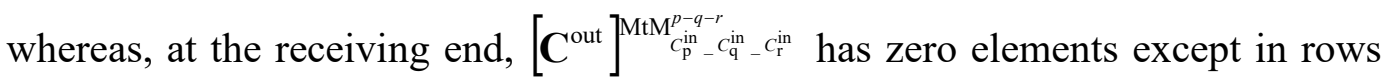
$p, q$, and $r$ where the values are equal to $\left|C_{p}^{\text {in }}\right| / C_{p}^{\text {in }},\left|C_{q}^{\text {in }}\right| / C_{q}^{\text {in }}$ and $\left|C_{r}^{\text {in }}\right| / C_{r}^{\text {in }}$, respectively.

\section{Broadband Performance Statistical Metrics and Regression Approximations of Distribution BPL Networks}

In this Section, the broadband performance statistical metrics of [17], which have been applied for the broadband performance assessment of MIMO channels of distribution BPL networks, are here modified in order to assess the broadband performance of various coupling schemes. In fact, the main modification is focused on the replacement of UVD modal channel transfer functions of [17] with the coupling scheme transfer function of eq. (1) as demonstrated in the following analysis.

More particularly, the broadband performance statistical metrics, which are applied in this paper, are the ACG, the RMS-DS, the CB and the SE while the 
computation of the discrete impulse response of the coupling scheme transfer function is considered as a prerequisite task. More specifically:

a. The discrete impulse response. Once the coupling scheme channel transfer function $H^{\mathrm{C}}\{\cdot\}$ has already been determined in eq. (1), the discrete coupling scheme channel transfer function $H^{\mathrm{C}+}$ that is an important transformation during the computation of broadband performance metrics is given by

$$
H^{C+}=\left\{\begin{array}{ll}
\left|H_{q}^{C+}\right| e^{j \varphi_{q}^{+}}, & q=0, \ldots, K-1 \\
0, & q=K, \ldots, J-1
\end{array}\right\}=\left\{\begin{array}{ll}
H_{q}^{C+}\left(f=q f_{s}\right), & q=0, \ldots, K-1 \\
0, & q=K, \ldots, J-1
\end{array}\right\}
$$
where $F_{\mathrm{s}}=1 / T_{\mathrm{s}}$ is the Nyquist sampling rate, $K \leq J / 2$ is the number of subchannels in the BPL signal frequency range of interest, $f_{\mathrm{s}}=F_{\mathrm{s}} / J$ is the flat-fading subchannel frequency spacing. $\left|H_{q}^{C+}\right|$ and $\varphi_{q}^{+}, q=0, \ldots, J-1$ are the amplitude responses and the phase responses of the discrete coupling scheme channel transfer function, respectively [17], [41]-[43].

Discrete coupling scheme impulse response $h^{C+}=h^{C+}\left(t=p T_{\mathrm{s}}\right), p=0, \ldots, J-1$ is obtained as the power of two $J$-point Inverse Discrete Fourier Transform (IDFT) of the discrete coupling scheme channel transfer function of eq. (3).

b. ACG. ACGs of coupling scheme channels is computed by averaging over frequency [17], [41]-[47]:

$$
\overline{\left|H^{C+}\right|^{2}}=\sum_{p=0}^{J-1}\left|h_{p}^{C+}\right|^{2}=\frac{1}{J} \sum_{q=0}^{J-1}\left|H_{q}^{C+}\right|^{2}
$$

c. RMS-DS. The RMS-DS of coupling scheme channels $\sigma_{\tau}^{C+}$ is determined from [17], [41]-[47]:

$$
\sigma_{\tau}^{C+}=T_{\mathrm{s}} \sqrt{\mu_{0}^{(2)}-\left(\mu_{0}\right)^{2}}
$$

where

$$
\begin{gathered}
\mu_{0}=\frac{\sum_{p=0}^{J-1} p\left|h_{p}^{C+}\right|^{2}}{\sum_{p=0}^{J-1}\left|h_{p}^{C+}\right|^{2}} \\
\mu_{0}^{(2)}=\frac{\sum_{p=0}^{J-1} p^{2}\left|h_{p}^{C+}\right|^{2}}{\sum_{p=0}^{J-1}\left|h_{p}^{C+}\right|^{2}}
\end{gathered}
$$

d. $C B$. It is the range of frequencies over which the normalized autocorrelation function of the channel transfer function is over a certain CB correlation level $X$ (usually set to either 0.9 or 0.7 or 0.5 ), i.e., the maximum bandwidth in which the subchannels can be approximately considered flat-fading. In accordance with [17], the $\mathrm{CB}$ of coupling scheme channels can be determined by:

$$
B_{\mathrm{c}}(\Delta f)=\frac{\mathrm{E}\left\{H_{q}^{C+} \cdot\left[H^{C+}\left(f=q f_{\mathrm{s}}+\Delta f\right)\right]^{*}\right\}}{\mathrm{E}\left\{\left.H_{q}^{C+}\right|^{2}\right\}}, q=0,1, \ldots,\left\lfloor(J-2)+\frac{\Delta f}{f_{s}}\right\rfloor
$$

where $\Delta f$ is the frequency shift, $[\cdot]^{*}$ denotes the complex conjugate of an element and $\lfloor x\rfloor$ is the largest integer not greater than $x$. From eq. (8), $C B_{X}$ is that value of $\Delta f$ such that $B_{\mathrm{c}}(\Delta f)=X$ while $C B_{0.5}$ is used in this paper. 
e. Capacity and SE. In accordance with [33], capacity is defined as the maximum achievable transmission rate that can be reliably transmitted over a BPL topology. Capacity depends on the applied MTL configuration, the BPL topology, the coupling scheme applied, EMI policies adopted and the noise environment.

The capacity $C$ for given coupling scheme channel is determined from

$$
C=f_{s} \sum_{q=0}^{K-1} \log _{2}\left\{1+\left[\frac{\left\langle p\left(q \cdot f_{s}\right)\right\rangle_{L}}{\left\langle N\left(q \cdot f_{s}\right)\right\rangle_{L}} \cdot \mid H^{\mathrm{C}+}\left(q \cdot f_{s}\right)^{2}\right]\right\}
$$

where $\langle\cdot\rangle_{L}$ is an operator that converts $\mathrm{dBm} / \mathrm{Hz}$ into a linear power ratio $(\mathrm{W} / \mathrm{Hz})$, $p(f)$ is the injected power spectral density limits (IPSD limits) and $N(f)$ is the uniform additive white Gaussian noise (AWGN) PSD level.

Finally, SE is based on the previous capacity computations and refers to the information in bps $/ \mathrm{Hz}$ that can be reliably transmitted over the used BPL bandwidth for the examined distribution BPL topology. Since capacity computations of this paper are given in $3-88 \mathrm{MHz}$ frequency range, spectral efficiency is given by

$$
S E=\frac{C}{85 \mathrm{MHz}}
$$

In accordance with [33], the IPSD limits that are applied are proposed by Ofcom in order to provide a presumption of compliance with FCC Part 15. However, these IPSD limits depend on the type of the distribution power grid (i.e., either $\mathrm{OV}$ or $\mathrm{UN}$ ) and the $\mathrm{BPL}$ frequency range (i.e., either $3-30 \mathrm{MHz}$ or $30-88 \mathrm{MHz}$ ). Hence, for given distribution power grid type, the average SE is given by eq. (10) since the capacity computations consider the $3-88 \mathrm{MHz}$ frequency range.

Apart from the broadband performance statistical metrics, three regression approaches (i.e., UN1, UN2 and UN3 approach) are applied and computed in this paper in order to describe the behavior of coupling scheme BPL channels. Among the three regression approaches, the first two ones have been introduced in [17] while the last one is first presented in this paper. More specifically:

- UN1 approach. UN1 approach linearly approximates the negatively correlated lognormal relation between ACG and RMS-DS of coupling scheme channels of distribution BPL topologies. In this paper, the regression approximation of UN1 approach is compared against the corresponding one of MIMO channels of distribution BPL topologies as demonstrated in [17].

- UN2 approach. UN2 approach describes the correlation relation between CB and RMS-DS of coupling scheme channels of distribution BPL topologies through suitable hyperbolic functions. The results of UN2 approach are compared against the respective ones of MIMO channels of distribution BPL topologies of [17]. Since these indicative BPL topologies are carefully selected so as to be the same with those of [17], UN1 and UN2 approaches of this paper concerning the coupling scheme channels are going to be compared against the respective ones of MIMO channels of [17].

- UN3 approach. UN3 approach demonstrates the correlation between SE and ACG. With reference to eq. (10), SE depends on the IPSD limits and AWGN PSD noise levels but also on the channel attenuation of the examined distribution BPL 
topologies. Here, the relation between SE and channel attenuation is clarified. Furthermore, the impact of the applied coupling scheme module (i.e., CS2 and CS1 module) on the UN3 approach is first investigated through its impact on the coupling transfer functions.

\section{Numerical Results and Discussion}

The simulations and respective numerical results of this Section aim at assessing the transmission performance of CS2 module against the vintage CS1 module. Extending the capacity observations of [33], the comparison between CS2 and CS1 module is based on the results of the broadband performance statistical metrics of ACG, RMS-DS, CB and SE. Also, the universal role of UN1 and UN2 approaches is examined in distribution BPL networks regardless of the coupling scheme module (i.e., CS2 or CS1 module) or the channel type (i.e., coupling scheme channel or MIMO channel). In addition, the behavior of UN3 approach is first presented and then investigated in terms of the applied coupling scheme module.

For the numerical computations, the indicative OV and UN distribution BPL topologies of Tables 1 and 2 (denoted hereafter as indicative topologies) are considered. With reference to eq. (1), the numerical results focus on the coupling transfer function from the application of CS2 and CS1 modules rather than on the behavior of the channel transfer function matrices since the last issue has thoroughly been analyzed in [3], [6], [11], [14]-[16], [33].

For comparison reasons, the same simulation model properties with [17] concerning the sampling and IDFT settings are here assumed. More specifically, the operating frequency band, the Nyquist sampling rate $F_{s}$ and flat-fading subchannel frequency spacing $f_{s}$ are assumed equal to $1-100 \mathrm{MHz}, 200 \mathrm{MHz}$ and $0.1 \mathrm{MHz}$, respectively. Therefore, the number of subchannels $K$ in the BPL signal frequency range of interest and the $J$-point IDFT are assumed equal to 991 and 2048, respectively [41]-[43].

As the capacity computation model properties are regarded, these are assumed to be the same with [17], [33], namely, the IPSD limits proposed by Ofcom are applied while AWGN PSD levels of $-105 \mathrm{dBm} / \mathrm{Hz}$ and $-135 \mathrm{dBm} / \mathrm{Hz}$ in the case of overhead and underground BPL networks, respectively, are applied in order to describe the noise conditions. For the sake of comparison, $3-88 \mathrm{MHz}$ frequency range is only assumed for the case of capacity and SE computations since the aforementioned IPSD limits are so defined.

Finally, as the properties of coupling schemes are concerned, representative coupling schemes of the three coupling scheme types of Sec. III are considered so that a clear transmission performance comparison between CS2 and CS1 module can be presented by simultaneously respecting the manuscript size limitations. Hence, the coupling schemes types of $\mathrm{WtG}^{1} / \mathrm{StP}^{1}, \mathrm{WtW}^{2-3} / \mathrm{PtP}^{2-3}$ and $\mathrm{MtM}_{0.7-0.1_{-}-0.2}^{1-2-3}$ are the representative ones for the coupling scheme type 1, 2 and 3, respectively. Already been mentioned, WtG and $\mathrm{WtW}$ coupling schemes are related to $\mathrm{OV}$ distribution $\mathrm{BPL}$ topologies whereas StP and PtP coupling schemes are related to UN distribution BPL topologies. MtM coupling schemes are common for both OV and UN distribution BPL topologies. Note that during MtM coupling schemes, the fourth conductor, where it exists, is not used. For the representative coupling schemes, the broadband performance 
statistical metrics as well as the regression approximations are applied so that their performance and the overall performance of CS2 module can be examined.

\subsection{ACG}

ACG is a statistical metric that describes the complexity of the examined BPL topologies. The lowest ACG values imply an intense multipath environment where high number of short branches exists. Also, ACG facilitates the comparison between CS2 and CS1 module since their impact during the injection and extraction process of BPL signal is clearly quantified.

In Table 3, ACG of the indicative OV LV and OV MV topologies is reported when CS2 and CS1 modules are considered and the aforementioned representative coupling schemes are applied. Same results with Table 3 are reported for the indicative UN LV and UN MV topologies in Table 4.

Table 3

ACG of the Indicative OV Distribution BPL Topologies for the Representative Coupling Schemes when CS2 and CS1 Modules are Considered

\begin{tabular}{|c|c|c|c|c|c|c|c|}
\hline & & \multicolumn{6}{|c|}{$\begin{array}{l}\mathrm{ACG} \\
(\mathrm{dB})\end{array}$} \\
\hline & & \multicolumn{2}{|c|}{$\mathrm{WtG}^{1}$} & \multicolumn{2}{|c|}{$\mathrm{WtW}^{2-3}$} & \multicolumn{2}{|c|}{$\mathrm{MtM}_{0.7_{-}-0.1_{-}-0.2}^{1-2-3}$} \\
\hline & & CS2 & CS1 & CS2 & CS1 & CS2 & CS1 \\
\hline \multirow[t]{2}{*}{ Urban case $A$} & LV & -9.53 & -9.53 & -9.40 & -15.42 & -9.05 & - \\
\hline & MV & -9.84 & -9.84 & -9.45 & -15.47 & -9.09 & - \\
\hline \multirow[t]{2}{*}{ Urban case B } & LV & -12.70 & -12.70 & -12.59 & -18.62 & -12.19 & - \\
\hline & MV & -13.01 & -13.01 & -12.54 & -18.56 & -12.14 & - \\
\hline \multirow[t]{2}{*}{ Suburban case } & LV & -8.22 & -8.22 & -8.08 & -14.10 & -7.72 & - \\
\hline & MV & -8.54 & -8.54 & -8.09 & -14.11 & -7.72 & - \\
\hline \multirow[t]{2}{*}{ Rural case } & LV & -6.74 & -6.74 & -6.54 & -12.56 & -6.12 & - \\
\hline & MV & -7.06 & -7.06 & -6.51 & --12.53 & -6.09 & - \\
\hline \multirow[t]{2}{*}{ "LOS" case } & LV & -4.65 & -4.65 & -4.51 & -10.53 & -4.17 & - \\
\hline & MV & -4.92 & -4.92 & -4.48 & -10.51 & -4.14 & - \\
\hline
\end{tabular}


Table 4

ACG of the Indicative UN Distribution BPL Topologies for the Representative Coupling Schemes when CS2 and CS1 Modules are Considered

\begin{tabular}{|c|c|c|c|c|c|c|c|}
\hline & & \multicolumn{6}{|c|}{$\begin{array}{l}\mathrm{ACG} \\
(\mathrm{dB})\end{array}$} \\
\hline & & \multicolumn{2}{|c|}{$\mathrm{StP}^{1}$} & \multicolumn{2}{|c|}{$\mathrm{PtP}^{2-3}$} & \multicolumn{2}{|c|}{$\mathrm{MtM}_{0.7_{-}-0.1_{-}-0.2}^{1-2-3}$} \\
\hline & & CS2 & CS1 & CS2 & CS1 & CS2 & CS1 \\
\hline \multirow[t]{2}{*}{ Urban case $\mathrm{A}$} & $\mathrm{LV}$ & -15.40 & -15.40 & -16.70 & -22.72 & -15.97 & - \\
\hline & $\mathrm{MV}$ & -25.14 & -25.14 & -25.14 & -31.16 & -25.14 & - \\
\hline \multirow[t]{2}{*}{ Urban case B } & LV & -19.18 & -19.18 & -20.47 & -26.49 & -19.74 & - \\
\hline & MV & -27.08 & -27.08 & -27.08 & -33.10 & -27.08 & - \\
\hline \multirow[t]{2}{*}{ Suburban case } & LV & -14.42 & -14.42 & -15.70 & -21.72 & -14.98 & - \\
\hline & $\mathrm{MV}$ & -23.31 & -23.31 & -23.31 & -29.33 & -23.31 & - \\
\hline \multirow[t]{2}{*}{ Rural case } & LV & -12.20 & -12.20 & -13.42 & -19.44 & -12.74 & - \\
\hline & $\mathrm{MV}$ & -20.78 & -20.78 & -20.78 & -26.80 & -20.78 & - \\
\hline \multirow[t]{2}{*}{ "LOS" case } & LV & -9.46 & -9.46 & -10.59 & -16.61 & -9.96 & - \\
\hline & MV & -17.95 & -17.95 & -17.95 & -23.97 & -17.95 & - \\
\hline
\end{tabular}

From Tables 3 and 4, several interesting remarks concerning the applied coupling schemes and examined coupling scheme modules as well as their interaction can be pointed out. First, ACGs of OV and UN LV and MV topologies remain the same for given BPL topology regardless of the $\mathrm{WtG}^{1}$ or $\mathrm{StP}^{1}$ coupling scheme applied. This is a rather logical observation for the coupling schemes of type 1 since $\left[\mathbf{C}^{\text {in }}\right]^{\mathrm{WtG} / \mathrm{StP}^{\mathrm{s}}}$ and $\left[\mathbf{C}^{\text {out }}\right]^{\mathrm{WtG}_{\mathrm{S}}^{\mathrm{s}} / \mathrm{StP}^{\mathrm{s}}}$ of CS2 module are equal to the respective coupling vectors of the vintage CS1 module. Anyway, the main differences of CS2 and CS1 module are focused on the coupling scheme type 2 and 3.

As the coupling schemes of type 2 are concerned, CS2 module achieves better ACGs than CS1 module since $\left[\mathbf{C}^{\text {out }}\right]^{\mathrm{WtW}^{p-q} / \mathrm{PtP}^{p-q}}$ of $\mathrm{CS} 2$ module allows the full reconstruction of the output signal in contrast with the half-signal reception of CS1 module due to $\left[\mathbf{C}^{\text {out }}\right]^{\mathrm{WtW}^{p-q} / \mathrm{PtP}^{p-q}}$. Hence, as it is expected, ACG difference between CS2 and CS1 module remains approximately equal to $+6 \mathrm{~dB}$ and this is numerically validated from the comparison of CS1 and CS2 columns of $\mathrm{WtW}^{2-3}$ and $\mathrm{PtP}^{2-3}$ coupling schemes in Table 3 and 4, respectively. Although CS2 module can significantly improve ACG of coupling schemes of type 2, WtW and PtP coupling schemes still present slightly worst ACG performance in comparison with the respective one of $\mathrm{WtG}$ and $\mathrm{StP}$ coupling schemes for given BPL network type.

As the coupling schemes of type 3 are examined, only CS2 module may support these coupling schemes due to its capability to discretely define $\left[\mathbf{C}^{\text {in }}\right]^{\mathrm{WtG^{ \textrm {s } }} / \mathrm{StP}^{\mathrm{s}}}$ and $\left[\mathbf{C}^{\text {out }}\right]^{\mathrm{WtG}^{\mathrm{s}} / \mathrm{StP}^{\mathrm{s}}}$. Despite its installation complexity, MtM coupling schemes exploit either the better ACG performance of coupling schemes of type 1 or the better electromagnetic compatibility (EMC) efficiency of coupling schemes of type 2. By appropriately

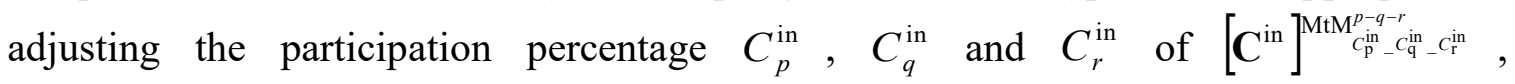


a trade-off relationship between broadband transmission, capacity performance and EMC can be adjusted through remote monitoring and surveillance.

\subsection{RMS-DS}

Already been mentioned in [17], RMS-DS is a highly variable statistical performance metric that depends on the power grid type and the examined BPL topology. Similarly to the ACG approach of Sec.5.1A, RMS-DS of the indicative OV LV and OV MV topologies is reported in Table 5 when CS2 and CS1 modules are considered and the aforementioned representative coupling schemes are applied. Same results with Table 5 are reported for the indicative UV LV and UN MV BPL topologies in Table 6.

Similarly to ACG, RMS-DS reflects the complexity of the examined BPL topology but, in contrast with ACG, RMS-DS cannot directly quantify the impact of the applied coupling scheme modules; RMS-DS values are the same for CS2 and CS1 module for given power grid type and BPL topology. However, RMS-DS computation remains critical since its values are necessary for the UN1 and UN2 approximations. In general, RMS-DS presents higher values as the BPL topology complexity increases; say, urban topologies present higher RMS-DS values than those of suburban, rural and "LOS" topologies due to the their aggravated multipath environment [48], [49].

Table 5

RMS-DS of the Indicative OV Distribution BPL Topologies for the Representative Coupling Schemes when CS2 and CS1 Modules are Considered

\begin{tabular}{|c|c|c|c|c|c|c|c|}
\hline & & \multicolumn{6}{|c|}{$\begin{array}{c}\text { RMS-DS } \\
(\mu \mathrm{s})\end{array}$} \\
\hline & & \multicolumn{2}{|c|}{$\mathrm{WtG}^{1}$} & \multicolumn{2}{|c|}{$\mathrm{WtW}^{2-3}$} & \multicolumn{2}{|c|}{$\mathrm{MtM}_{0.7_{-}-0.1_{-}-0.2}^{1-2-3}$} \\
\hline & & CS2 & CS1 & CS2 & CS1 & CS2 & CS1 \\
\hline \multirow[t]{2}{*}{ Urban case $A$} & LV & 0.56 & 0.56 & 0.59 & 0.59 & 0.61 & - \\
\hline & MV & 0.74 & 0.74 & 0.64 & 0.64 & 1.01 & - \\
\hline \multirow[t]{2}{*}{ Urban case B } & LV & 1.11 & 1.11 & 1.18 & 1.18 & 1.25 & - \\
\hline & MV & 1.07 & 1.07 & 1.18 & 1.18 & 1.28 & - \\
\hline \multirow[t]{2}{*}{ Suburban case } & LV & 0.53 & 0.53 & 0.55 & 0.55 & 0.58 & - \\
\hline & $\mathrm{MV}$ & 0.64 & 0.64 & 0.58 & 0.58 & 0.84 & - \\
\hline \multirow[t]{2}{*}{ Rural case } & LV & 1.07 & 1.07 & 1.09 & 1.09 & 1.12 & - \\
\hline & $\mathrm{MV}$ & 1.08 & 1.08 & 1.10 & 1.10 & 1.18 & - \\
\hline \multirow[t]{2}{*}{ "LOS" case } & LV & 0.09 & 0.09 & 0.10 & 0.10 & 0.10 & - \\
\hline & MV & 0.38 & 0.38 & 0.20 & 0.20 & 0.61 & - \\
\hline
\end{tabular}


Table 6

RMS-DS of the Indicative UN Distribution BPL Topologies for the Representative Coupling Schemes when CS2 and CS1 Modules are Considered

\begin{tabular}{|c|c|c|c|c|c|c|c|}
\hline & & \multicolumn{6}{|c|}{$\begin{array}{c}\text { RMS-DS } \\
(\mu \mathrm{s})\end{array}$} \\
\hline & & \multicolumn{2}{|c|}{$\mathrm{StP}^{1}$} & \multicolumn{2}{|c|}{$\mathrm{PtP}^{2-3}$} & \multicolumn{2}{|c|}{$\mathrm{MtM}_{0.7_{-}-0.1_{-}-0.2}^{1-2-3}$} \\
\hline & & CS2 & CS1 & CS2 & CS1 & CS2 & CS1 \\
\hline \multirow[t]{2}{*}{ Urban case $A$} & $\mathrm{LV}$ & 0.54 & 0.54 & 0.57 & 0.57 & 0.55 & - \\
\hline & MV & 1.21 & 1.21 & 1.21 & 1.21 & 1.21 & - \\
\hline \multirow[t]{2}{*}{ Urban case B } & LV & 0.83 & 0.83 & 0.84 & 0.87 & 0.85 & - \\
\hline & MV & 1.45 & 1.45 & 1.45 & 1.45 & 1.45 & - \\
\hline \multirow[t]{2}{*}{ Suburban case } & LV & 0.48 & 0.48 & 0.48 & 0.48 & 0.48 & - \\
\hline & MV & 0.79 & 0.79 & 0.79 & 0.79 & 0.79 & - \\
\hline \multirow[t]{2}{*}{ Rural case } & $\mathrm{LV}$ & 0.53 & 0.53 & 0.54 & 0.54 & 0.53 & - \\
\hline & MV & 0.87 & 0.87 & 0.87 & 0.87 & 0.87 & - \\
\hline \multirow[t]{2}{*}{ "LOS" case } & LV & 0.26 & 0.26 & 0.29 & 0.29 & 0.28 & - \\
\hline & MV & 0.60 & 0.60 & 0.60 & 0.60 & 0.60 & - \\
\hline
\end{tabular}

\section{$5.3 \mathrm{CB}$}

Similarly to RMS-DS, CB expresses the maximum bandwidth in which the subchannels can be approximately considered flat-fading in a BPL channel. $\mathrm{CB}_{0.5}$ of the indicative OV LV and OV MV topologies is reported in Table 7 when CS2 and CS1 modules are applied and the aforementioned representative coupling schemes are considered. Same results with Table 7 are reported for the indicative UV LV and UN MV BPL topologies in Table 8 .

Table 7

$\mathrm{CB}_{0.5}$ of the Indicative OV Distribution BPL Topologies for the Representative Coupling Schemes when CS2 and CS1 Modules are Considered

\begin{tabular}{|c|c|c|c|c|c|c|c|}
\hline & & \multicolumn{6}{|c|}{$\begin{array}{l}\mathrm{CB}_{0.5} \\
(\mathrm{MHz})\end{array}$} \\
\hline & & \multicolumn{2}{|c|}{$\mathrm{WtG}^{1}$} & \multicolumn{2}{|c|}{$\mathrm{WtW}^{2-3}$} & \multicolumn{2}{|c|}{$\mathrm{MtM}_{0.7_{-}-0.1_{-}-0.2}^{1-2-3}$} \\
\hline & & CS2 & CS1 & CS2 & CS1 & CS2 & CS1 \\
\hline \multirow[t]{2}{*}{ Urban case $\mathrm{A}$} & LV & 2.6 & 2.6 & 2.6 & 2.6 & 2.5 & - \\
\hline & MV & 2.7 & 2.7 & 2.6 & 2.6 & 2.4 & - \\
\hline \multirow[t]{2}{*}{ Urban case B } & LV & 0.7 & 0.7 & 0.7 & 0.7 & 0.7 & - \\
\hline & MV & 0.8 & 0.8 & 0.7 & 0.7 & 0.7 & - \\
\hline \multirow[t]{2}{*}{ Suburban case } & LV & 1.1 & 1.1 & 1.1 & 1.1 & 1.1 & - \\
\hline & MV & 1.1 & 1.1 & 1.1 & 1.1 & 1.1 & - \\
\hline \multirow[t]{2}{*}{ Rural case } & $\mathrm{LV}$ & 5.7 & 5.7 & 3.2 & 3.2 & 0.7 & - \\
\hline & MV & 6.7 & 6.7 & 3.2 & 3.2 & 0.2 & - \\
\hline \multirow[t]{2}{*}{ "LOS" case } & LV & 49.4 & 49.4 & 49.4 & 49.4 & 49 & - \\
\hline & MV & 47.6 & 47.6 & 49.3 & 49.3 & 46.6 & - \\
\hline
\end{tabular}


Table 8

$\mathrm{CB}_{0.5}$ of the Indicative UN Distribution BPL Topologies for the Representative Coupling Schemes when CS2 and CS1 Modules are Considered

\begin{tabular}{|c|c|c|c|c|c|c|c|}
\hline & & \multicolumn{6}{|c|}{$\begin{array}{l}\mathrm{CB}_{0.5} \\
(\mathrm{MHz})\end{array}$} \\
\hline & & \multicolumn{2}{|c|}{$\mathrm{StP}^{1}$} & \multicolumn{2}{|c|}{$\mathrm{PtP}^{2-3}$} & \multicolumn{2}{|c|}{$\mathrm{MtM}_{0.7_{-}-0.1_{-}-0.2}^{1-2-3}$} \\
\hline & & CS2 & CS1 & CS2 & CS1 & CS2 & CS1 \\
\hline \multirow[t]{2}{*}{ Urban case A } & $\mathrm{LV}$ & 1.3 & 1.3 & 1.3 & 1.3 & 1.3 & - \\
\hline & MV & 0.6 & 0.6 & 0.6 & 0.6 & 0.6 & - \\
\hline \multirow[t]{2}{*}{ Urban case B } & $\mathrm{LV}$ & 0.8 & 0.8 & 0.7 & 0.7 & 0.7 & - \\
\hline & MV & 0.5 & 0.5 & 0.5 & 0.5 & 0.5 & - \\
\hline \multirow[t]{2}{*}{ Suburban case } & $\mathrm{LV}$ & 1.3 & 1.3 & 1.3 & 1.3 & 1.3 & - \\
\hline & MV & 1.1 & 1.1 & 1.1 & 1.1 & 1.1 & - \\
\hline \multirow[t]{2}{*}{ Rural case } & LV & 21.2 & 21.2 & 21.2 & 21.2 & 21.2 & - \\
\hline & MV & 3.8 & 3.8 & 3.8 & 3.8 & 3.8 & - \\
\hline \multirow[t]{2}{*}{ "LOS" case } & LV & 37 & 37 & 33.5 & 33.5 & 35.6 & - \\
\hline & MV & 5.8 & 5.8 & 5.8 & 5.8 & 5.8 & - \\
\hline
\end{tabular}

Similarly to ACG and RMS-DS, CB depends on the complexity of the examined BPL topology. BPL topologies with aggravated multipath environments require shorter subchannel bandwidths so that their channels can be considered as flat-fading ones. Numerically, $49.4 \mathrm{MHz}$ and $0.5 \mathrm{MHz}$ are the maximum and minimum flat-fading subchannel bandwidths that are reported in Tables 7 and 8 for the examined representative BPL topologies. Here, it should be noted that flat-fading subchannel frequency spacing $f_{s}$ is assumed equal to $0.1 \mathrm{MHz}$, thus allowing the flat-fading subchannel frequency consideration either during the computation of the statistical broadband performance metrics of this paper or the capacity computations of [33].

\subsection{Capacity and SE}

The behavior of the transmission performance metrics of ACG, RMS-DS and CB, which has been highlighted in the previous subsections, is also reflected on the capacity performance metrics. With reference to [33], capacity of the indicative OV LV and OV MV topologies is reported in Table 9 when CS2 and CS1 modules are applied and the aforementioned representative coupling schemes are considered. Here it should be reminded that both capacity performance metrics are computed in the $3-88 \mathrm{MHz}$ frequency range due to the frequency range limitations of the applied IPSD limits. Same results with Table 9 are reported for the indicative UV LV and UN MV BPL topologies in Table 10. In Table 11 and 12, SE values are computed for the same cases of Table 9 and 10 , respectively. 
Table 9

Capacity of the Indicative OV Distribution BPL Topologies for the Representative Coupling Schemes when CS2 and CS1 Modules are Considered

\begin{tabular}{|c|c|c|c|c|c|c|c|}
\hline & & \multicolumn{6}{|c|}{$\begin{array}{c}\text { Capacity } \\
\text { (Mbps) }\end{array}$} \\
\hline & & \multicolumn{2}{|c|}{$\mathrm{WtG}^{1}$} & \multicolumn{2}{|c|}{$\mathrm{WtW}^{2-3}$} & \multicolumn{2}{|c|}{$\mathrm{MtM}_{0.7_{-}-0.1_{-}-0.2}^{1-2-3}$} \\
\hline & & CS2 & CS1 & CS2 & CS1 & CS2 & CS1 \\
\hline \multirow[t]{2}{*}{ Urban case A } & $\mathrm{LV}$ & 606 & 606 & 612 & 463 & 620 & - \\
\hline & MV & 596 & 596 & 612 & 463 & 622 & - \\
\hline \multirow[t]{2}{*}{ Urban case B } & LV & 469 & 469 & 475 & 346 & 484 & - \\
\hline & MV & 459 & 459 & 476 & 346 & 485 & - \\
\hline \multirow[t]{2}{*}{ Suburban case } & LV & 715 & 715 & 721 & 561 & 730 & - \\
\hline & MV & 705 & 705 & 722 & 562 & 732 & - \\
\hline \multirow[t]{2}{*}{ Rural case } & LV & 797 & 797 & 803 & 637 & 811 & - \\
\hline & MV & 787 & 787 & 803 & 638 & 811 & - \\
\hline \multirow[t]{2}{*}{ "LOS" case } & LV & 902 & 902 & 908 & 738 & 916 & - \\
\hline & MV & 892 & 892 & 909 & 739 & 918 & - \\
\hline
\end{tabular}

Table 10

Capacity of the Indicative UN Distribution BPL Topologies for the Representative Coupling Schemes when CS2 and CS1 Modules are Considered

\begin{tabular}{|c|c|c|c|c|c|c|c|}
\hline & & \multicolumn{6}{|c|}{$\begin{array}{c}\text { Capacity } \\
\text { (Mbps) }\end{array}$} \\
\hline & & \multicolumn{2}{|c|}{$\mathrm{StP}^{1}$} & \multicolumn{2}{|c|}{$\mathrm{PtP}^{2-3}$} & \multicolumn{2}{|c|}{$\mathrm{MtM}_{0.7_{-}-0.1_{-}-0.2}^{1-2-3}$} \\
\hline & & CS2 & CS1 & CS2 & CS1 & CS2 & CS1 \\
\hline \multirow[t]{2}{*}{ Urban case $\mathrm{A}$} & LV & 1849 & 1849 & 1805 & 1635 & 1830 & - \\
\hline & MV & 815 & 815 & 815 & 698 & 815 & - \\
\hline \multirow[t]{2}{*}{ Urban case B } & LV & 1634 & 1634 & 1590 & 1420 & 1615 & - \\
\hline & MV & 685 & 685 & 685 & 581 & 685 & - \\
\hline \multirow[t]{2}{*}{ Suburban case } & $\mathrm{LV}$ & 1953 & 1953 & 1909 & 1739 & 1934 & - \\
\hline & MV & 890 & 890 & 890 & 767 & 890 & - \\
\hline \multirow[t]{2}{*}{ Rural case } & LV & 2053 & 2053 & 2008 & 1838 & 2034 & - \\
\hline & MV & 968 & 968 & 968 & 838 & 968 & - \\
\hline \multirow[t]{2}{*}{ "LOS" case } & LV & 2152 & 2152 & 2108 & 1938 & 2133 & - \\
\hline & MV & 1049 & 1049 & 1049 & 913 & 1049 & - \\
\hline
\end{tabular}


Table 11

SE of the Indicative OV Distribution BPL Topologies for the Representative Coupling Schemes when CS2 and CS1 Modules are Considered

\begin{tabular}{|c|c|c|c|c|c|c|c|}
\hline & & \multicolumn{6}{|c|}{$\begin{array}{c}\mathrm{SE} \\
(\mathrm{bps} / \mathrm{Hz}) \\
\end{array}$} \\
\hline & & \multicolumn{2}{|c|}{$\mathrm{WtG}^{1}$} & \multicolumn{2}{|c|}{$\mathrm{WtW}^{2-3}$} & \multicolumn{2}{|c|}{$\mathrm{MtM}_{0.7_{-}-0.1_{-}-0.2}^{1-2-2}$} \\
\hline & & CS2 & CS1 & CS2 & CS1 & CS2 & CS1 \\
\hline \multirow[t]{2}{*}{ Urban case $\mathrm{A}$} & $\mathrm{LV}$ & 7.13 & 7.13 & 7.20 & 5.45 & 7.29 & - \\
\hline & MV & 7.01 & 7.01 & 7.20 & 5.45 & 7.32 & - \\
\hline \multirow[t]{2}{*}{ Urban case B } & LV & 5.52 & 5.52 & 5.59 & 4.07 & 5.69 & - \\
\hline & MV & 5.40 & 5.40 & 5.60 & 4.07 & 5.71 & - \\
\hline \multirow[t]{2}{*}{ Suburban case } & $\mathrm{LV}$ & 8.41 & 8.41 & 8.48 & 6.60 & 8.59 & - \\
\hline & MV & 8.29 & 8.29 & 8.49 & 6.61 & 8.61 & - \\
\hline \multirow[t]{2}{*}{ Rural case } & $\mathrm{LV}$ & 9.38 & 9.38 & 9.45 & 7.49 & 9.54 & - \\
\hline & MV & 9.26 & 9.26 & 9.45 & 7.51 & 9.54 & - \\
\hline \multirow[t]{2}{*}{ "LOS" case } & $\mathrm{LV}$ & 10.61 & 10.61 & 10.68 & 8.68 & 10.78 & - \\
\hline & MV & 10.49 & 10.49 & 10.69 & 8.69 & 10.80 & - \\
\hline
\end{tabular}

Table 12

SE of the Indicative UN Distribution BPL Topologies for the Representative Coupling Schemes when CS2 and CS1 Modules are Considered

\begin{tabular}{|c|c|c|c|c|c|c|c|}
\hline & & \multicolumn{6}{|c|}{$\begin{array}{c}\mathrm{SE} \\
\text { (bps/Hz) }\end{array}$} \\
\hline & & \multicolumn{2}{|c|}{$\mathrm{StP}^{1}$} & \multicolumn{2}{|c|}{$\mathrm{PtP}^{2-3}$} & \multicolumn{2}{|c|}{$\mathrm{MtM}_{0.7_{-}-0.1_{-}-0.2}^{1-2-2}$} \\
\hline & & CS2 & CS1 & CS2 & CS1 & CS2 & CS1 \\
\hline \multirow[t]{2}{*}{ Urban case A } & $\mathrm{LV}$ & 21.75 & 21.75 & 21.24 & 19.24 & 21.53 & - \\
\hline & MV & 9.59 & 9.59 & 9.59 & 8.21 & 9.59 & - \\
\hline \multirow[t]{2}{*}{ Urban case B } & LV & 19.22 & 19.22 & 18.71 & 16.71 & 19.00 & - \\
\hline & MV & 8.06 & 8.06 & 8.06 & 6.84 & 8.06 & - \\
\hline \multirow[t]{2}{*}{ Suburban case } & $\mathrm{LV}$ & 22.98 & 22.98 & 22.46 & 20.46 & 22.75 & - \\
\hline & MV & 10.47 & 10.47 & 10.47 & 9.02 & 10.47 & - \\
\hline \multirow[t]{2}{*}{ Rural case } & $\mathrm{LV}$ & 24.15 & 24.15 & 23.62 & 21.62 & 23.93 & - \\
\hline & MV & 11.39 & 11.39 & 11.39 & 9.86 & 11.39 & - \\
\hline \multirow[t]{2}{*}{ "LOS" case } & LV & 25.32 & 25.32 & 24.80 & 22.80 & 25.09 & - \\
\hline & MV & 12.34 & 12.34 & 12.34 & 10.74 & 12.34 & - \\
\hline
\end{tabular}


From Tables 9-12, it is obvious that CS2 module achieves better spectral exploitation of the allocated BPL frequency band in comparison with the CS1 module in coupling schemes of type 2 and 3 . Only by appropriately adjusting the way that the BPL signal power is extracted at the receiving end, average SE improvement of $1.821 \mathrm{bps} / \mathrm{Hz}$ and $1.718 \mathrm{bps} / \mathrm{Hz}$ is achieved in $\mathrm{OV} / \mathrm{WtW}$ and $\mathrm{UN} / \mathrm{PtP}$ distribution BPL networks, respectively, while the respective capacity improvement is equal to $155 \mathrm{Mbps}$ and 146Mbps.

Apart from their special transmission metric attributes, MtM coupling schemes present notable capacity characteristics that can be combined with their EMC adaptability [33]. Here, it should be underlined that only CS2 module supports MtM coupling schemes in distribution BPL networks. Numerically, with reference to Table 11, an average MtM coupling scheme achieves better SE in all the indicative topologies of the OV distribution BPL networks examined. With reference to Table 12, the same average MtM coupling scheme achieves better SE behavior in the vast majority of the UN/PtP distribution BPL topologies examined while its values are comparable even to the ones of the UN/StP distribution BPL topologies. Therefore, MtM can combine the favourable characteristics of both coupling scheme types 1 and 2; say the high SE of coupling scheme type 1 and the EMC strength of coupling scheme type 2. However, the main disadvantage of MtM coupling schemes is their high cost due to the installation of additional BPL repeaters on the distribution OV and UN conductors.

Finally, as the benchmark SE performance of CS2 and CS1 module is concerned, when the coupling schemes of the coupling scheme type 1 are applied, capacity and SE improvements are not observed regardless of the adoption of CS2 or CS1 module.

\subsection{UN1 Approach}

In [17], the correlation between RMS-DS and ACG has been highlighted with respect to the OV and UN MIMO/LV and MIMO/MV BPL channels, say CC and XC of $\mathrm{OV}$ and UN LV and MV BPL topologies. Following this fundamental property of several wireline networks, such as DSL and coaxial ones, regression trend lines of the form $\left(\sigma_{\tau}\right)_{\mu s}=-v \cdot\left(\overline{|H|^{2}}\right)_{d B}+w$ have been used in the aforementioned MIMO BPL channels where $\left(\sigma_{\tau}\right)_{\mu s}$ is the RMS-DS in $\mu$ s of the examined BPL channels, $\left(\overline{|H|^{2}}\right)_{d B}$ is the ACG in $\mathrm{dB}$ and $v$ and $w$ are the robust regression parameters. $\mathrm{UN} 1_{\mathrm{MIMO}}$ approach, which has been proposed in [17], is characterized by a set of robust regression parameters $\mathrm{v}$ and $\mathrm{w}$ that comes from the least squares fitting method being applied to the ACG / RMS-DS values of MIMO BPL channels regardless of the power grid type, BPL topology and MIMO channel type.

RMS-DS / ACG regression curves, which come from the linear regression approximations of different measurement campaign data, have been extensively proposed in the BPL literature for various BPL network types (e.g., in-home, in-ship, in-car, OV LV and OV MV BPL networks) [41], [50]-[53]. UN1 approach is compared against other well validated approximations of the BPL literature that deals with the coupling channels of various BPL networks; say, ANT approach and GAL approach as given by [46] and [41]-[43], respectively. Analytically, the robust regression parameters of each approach are reported in Table 13. In the same Table, the robust regression parameters $v$ and $w$ of 
$\mathrm{UN1} 1_{\mathrm{CS} 2}$ approach are computed for all the BPL topologies regardless of the power grid type when $\mathrm{WtG}^{1}, \mathrm{StP}^{1}, \mathrm{WtW}^{2-3}, \mathrm{PtP}^{2-3}$ and $\mathrm{MtM}_{0.7-0.1-0.2}^{1-2-3}$ of $\mathrm{CS} 2$ module are applied in accordance with Tables 3-6. Similarly to $\mathrm{UN1} 1_{\mathrm{CS} 2}$ approach, the robust regression parameters $v$ and $w$ of $\mathrm{UN1}_{\mathrm{CS} 1}$ approach are computed for all the BPL topologies regardless of the power grid type when $\mathrm{WtG}^{1}, \mathrm{StP}^{1}, \mathrm{WtW}^{2-3}$ and $\mathrm{PtP}^{2-3}$ of CS1 module are applied in accordance with Tables 3-6.

In Fig. 3, except for the simulation data of Tables 3-6, the set of regression trend

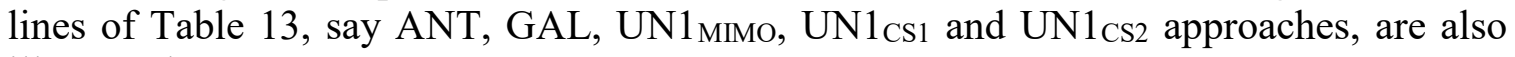
illustrated.

Table 13

Robust Regression Parameters $v$ and $w$ of the Different RMS-DS / ACG Approaches

\begin{tabular}{|c|c|c||c||c||c|}
\hline & ANT & GAL & UN1 $_{\text {MIMO }}$ & UN1 $_{\text {CS1 }}$ & $\mathrm{UN}_{\text {CS2 }}$ \\
\hline $\begin{array}{c}v \\
(\mu \mathrm{s} / \mathrm{dB})\end{array}$ & -0.0197 & -0.0075 & -0.01029 & -0.0227 & -0.0242 \\
\hline $\begin{array}{c}w \\
(\mu \mathrm{s})\end{array}$ & 0 & 0.183 & 0.59 & 0.3659 & 0.4378 \\
\hline
\end{tabular}

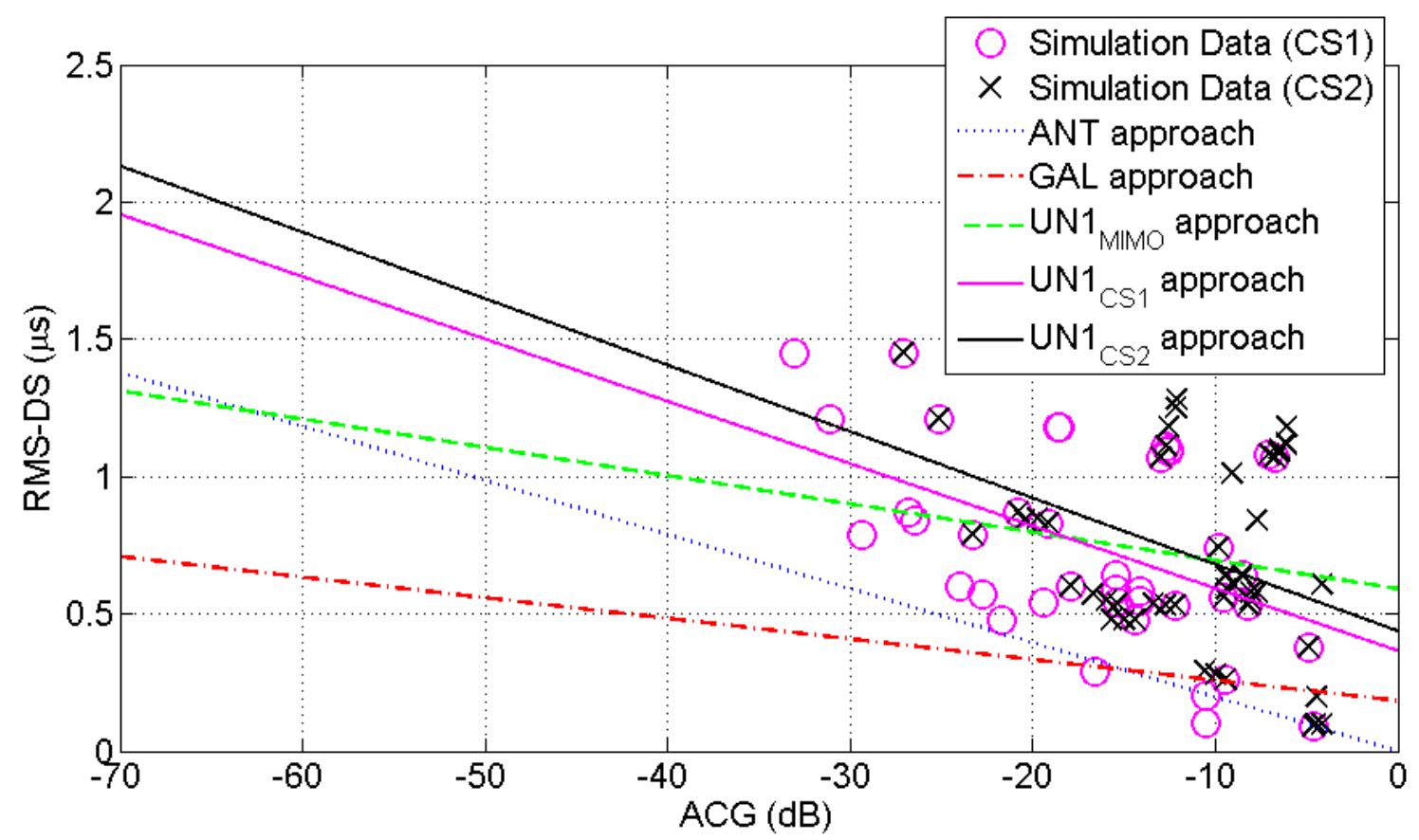

Fig. 3. Scatter plot of RMS-DS versus ACG for simulated coupling scheme OV and UN MV and LV BPL channels and various regression approaches. 
From Fig. 3, several interesting remarks concerning the performance of regression approximations can be pointed out, namely:

- Either MIMO or coupling scheme channels are examined, ACG and RMS-DS of $\mathrm{OV}$ and UN LV and MV BPL channels are negatively correlated lognormal random variables. This fact is validated by the negative slopes of all regression lines that approximate simulation data in Fig. 3.

- The set of UN1 regression lines, say UN1 $1_{\text {MIMO, }} \mathrm{UN1} 1_{\mathrm{CS} 1}$ and $\mathrm{UN} 1_{\mathrm{CS} 2}$ approaches, better approximates the simulation in comparison with ANT and GAL approaches. This is due to the fact that the set of UN1 regression lines is based on simulation data of distribution BPL networks rather on other various types of BPL networks (indoor, in-vehicle, etc).

- The differences among UN1 regression lines remain low enhancing the role of UN1 regression lines as a unified regression approach [17]. More specifically:

- The small differences between UN1MIMO approach and UN1 $1_{\text {CSx }}$ approaches, $x=1,2$ are due to the different origin of the processed simulation data; the former approach is based on the approximation of MIMO channels while the latter approaches approximate simulation data of coupling scheme channels. Hence, the impact of signal coupling is reflected on the differences of the aforementioned approaches.

- The differences between $\mathrm{UN} 1_{\mathrm{CS} 1}$ and $\mathrm{UN} 1_{\mathrm{CS} 2}$ approaches can be considered as negligible while they come from the versatility of coupling scheme types supported by CS2 module. In comparison with the supported coupling schemes by CS1 module, CS2 module mainly affects the performance of coupling schemes of type 2, i.e., WtW and PtP coupling schemes, while coupling schemes of type 3, i.e., MtM coupling schemes, are only supported by CS2 module. Since coupling schemes of type 1 remain the same, the difference between $\mathrm{UN} 1_{\mathrm{CS} 1}$ and $\mathrm{UN} 1_{\mathrm{CS} 2}$ approaches can be considered as the weighted mean average of the supported coupling scheme types; for example, if RMS-DS is assumed to be equal to $1 \mu \mathrm{s}$, the difference between the AVG of UN1CS1 and UN1CS2 approach is equal to $4.7 \mathrm{~dB}$, that is near to the two thirds of the $+6 \mathrm{~dB}$ ACG difference between CS2 and CS1 module mentioned in Sec.VA where two thirds corresponds to the number of coupling scheme types affected by CS2 module.

\subsection{UN2 Approach}

UN2 approach describes the fundamental correlation between CB and RMS-DS. In [17], this fundamental property of OV and UN LV and MV MIMO BPL channels has been reported and has been described through an appropriate hyperbolic trend curves set while the efficiency of UN2MIMO approach has been computed with respect to suitable simulation data of MIMO channels.

Similarly to RMS-DS / ACG regression curves, RMS-DS / CB hyperbolic trend curves have widely been used in various BPL network types [50], [52], [53]. In fact, the regression hyperbolic trend curves of the form $\left(\sigma_{\tau}\right)_{\mu \mathrm{s}}=y \cdot\left(C B_{0.5}\right)_{\mathrm{MHz}}^{-1}$ are also used in $\mathrm{UN} 2$ approach where $y$ is the robust regression parameter. In this paper, the same form of regression hyperbolic trend curves are going to be used for the assessment of coupling 
scheme BPL channels when CS2 and CS1 modules are adopted. By using robust linear least square error fitting between the trend and the simulation data of the OV and UN LV and MV BPL coupling scheme channels as reported in Tables 5-8, the parameter y of corresponding $\mathrm{UN} 2 \mathrm{CS} 1$ and $\mathrm{UN} 2 \mathrm{CS}_{2}$ approaches can be calculated. Analytically, the robust regression parameter y of each approach is reported in Table 11.

In Fig. 4, except for the simulation data of Tables 5-8, the set of regression trend curves of Table 10, say UN2 ${ }_{\mathrm{MIMO}}, \mathrm{UN} 2_{\mathrm{CS} 1}$ and UN2 ${ }_{\mathrm{CS} 2}$ approaches, are illustrated.

Table 14

Robust Regression Parameter $y$ of the Different RMS-DS / $\mathrm{CB}_{0.5}$ Approaches

\begin{tabular}{|c|c||c||c|}
\hline & $\mathrm{UN}_{\text {MIMO }}$ & $\mathrm{UN}_{\mathrm{CS} 1}$ & $\mathrm{UN}{ }_{\mathrm{CS} 2}$ \\
\hline $\begin{array}{c}y \\
(\mu \mathrm{s} \cdot \mathrm{MHz})\end{array}$ & 0.4155 & 0.7912 & 0.6131 \\
\hline
\end{tabular}

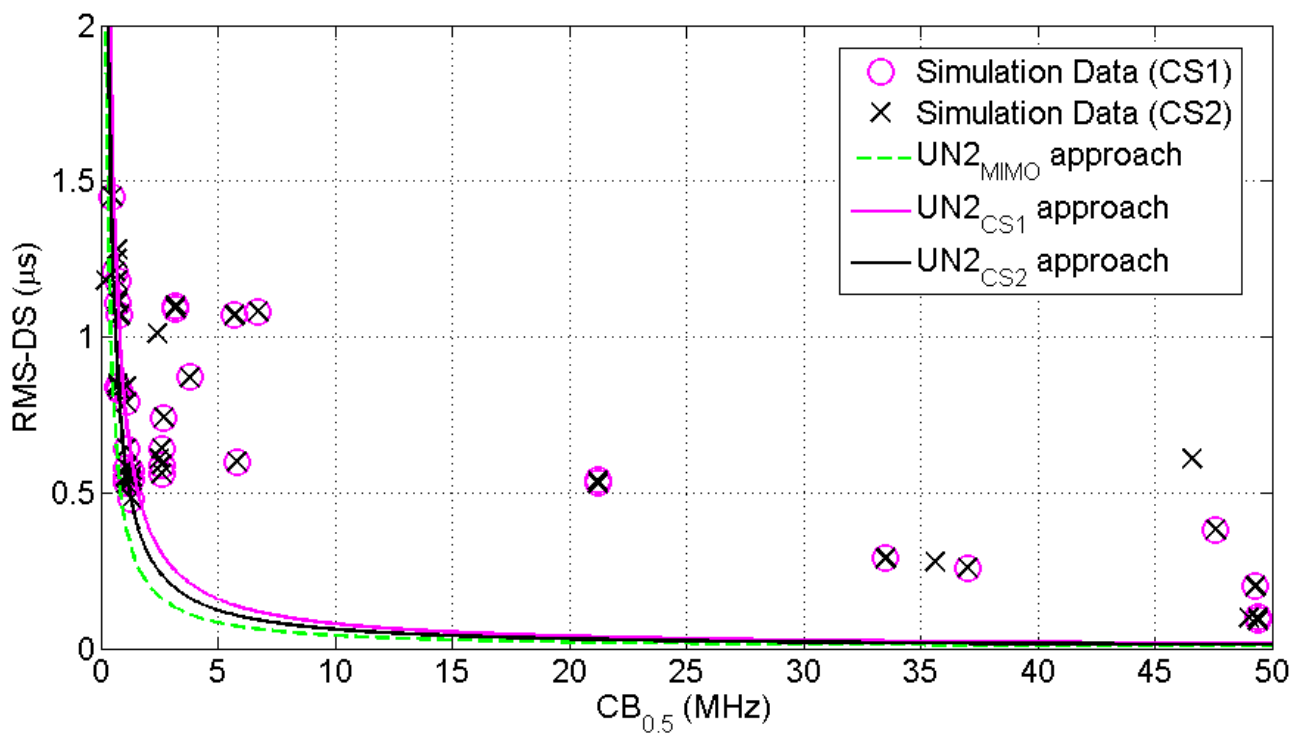

Fig. 4. Scatter plot of RMS-DS versus CB0.5 for simulated coupling scheme OV and UN LV and MV BPL channels and various regression approaches.

From Fig. 4, additional observations regarding the performance of regression approximations can be given, namely:

- $\mathrm{CB}$ and RMS-DS remain inversely related each other either in MIMO or in coupling scheme channels of distribution BPL networks. In both cases, appropriate hyperbolic functions can approximate $\mathrm{CB}$ and RMS-DS simulation data.

- Differences among UN2 ${ }_{\mathrm{MIMO}}, \mathrm{UN} 2_{\mathrm{CS} 1}$ and $\mathrm{UN} 2_{\mathrm{CS} 2}$ approaches remain marginal despite the high variability of CB / RMS-DS simulation. The main divergences of UN2 approach curves are focused on the rural and "LOS" topologies of 
distribution BPL networks whose RMS-DSs remain high due to the long average path lengths of distribution BPL networks.

- Due to the low differences of UN2 ${ }_{\text {MIMO, }} \mathrm{UN} 2{ }_{\mathrm{CS} 1}$ and $\mathrm{UN} 2{ }_{\mathrm{CS}}$ approaches, each of the UN2 approaches can sustain a unified consideration of the CB / RMS-DS correlation of either MIMO or coupling scheme channels.

\subsection{UN3 Approach}

UN3 approach, which is first presented in this paper, achieves to correlate the average SE and ACG of a distribution BPL topology. With respect to eq. (9), capacity significantly depends on the channel attenuation of the examined distribution BPL topology across the frequency range of interest but not only on this. In this paper, the different coupling scheme modules that are benchmarked do influence the coupling transfer function and, thus, its ACG. In order to highlight: (i) the SE dependence on the ACG when IPSD limits and noise PSD levels are assumed to be common; (ii) the role of the coupling scheme modules; and (iii) the SE dependence diversification on the IPSD limits and noise PSD levels when OV and UN distribution BPL networks are assumed, UN3 approach is here proposed following the definition of UN1 approach. In this subsection, UN3 approach examines the aforementioned correlation when CS2 and CS1 modules are applied.

UN3 approach is assumed to be described by regression trend lines of the form $(S E)_{\mathrm{bps} / \mathrm{Hz}}=-a \cdot\left(\overline{|H|^{2}}\right)_{d B}+\beta$ where $(S E)_{\mathrm{bps} / \mathrm{Hz}}$ is the average SE given in Tables 11 and 12 for $\mathrm{OV}$ and UN distribution BPL networks, respectively, while $a$ and $\beta$ are the robust regression parameters that come from the least squares fitting method. In order to investigate the SE performance diversification due to the different IPSD limits and noise PSD levels that are used in OV and UN distribution networks, two different regression trend lines of UN3 approach are presented for each coupling scheme module; say, one regression trend line for the $\mathrm{OV}$ distribution networks (i.e., $\mathrm{UN} 3^{\mathrm{OV}}$ approach) and another one for the $\mathrm{UN}$ distribution networks (i.e., $\mathrm{UN} 3{ }^{\mathrm{UN}}$ approach). Analytically, the robust regression parameters of each approach per coupling scheme module are reported in Table 15. Note that $a$ and $\beta$ of each column of Table 15 are computed for all

Table 15

Robust Regression Parameter $\alpha$ and $\beta$ of the Different SE / ACG Approaches

\begin{tabular}{|c|c|c||c||c|}
\hline & $\mathrm{UN} 3_{\mathrm{CS} 1-\mathrm{OV}}$ & $\mathrm{UN} 3_{\mathrm{CS} 1-\mathrm{UN}}$ & $\mathrm{UN} 3_{\mathrm{CS} 2-\mathrm{OV}}$ & $\mathrm{UN} 3_{\mathrm{CS} 2-\mathrm{UN}}$ \\
\hline $\begin{array}{c}\alpha \\
\mathrm{bps} /(\mathrm{dB} \cdot \mathrm{Hz})\end{array}$ & 0.4472 & 0.8627 & 0.6434 & 1.1154 \\
\hline $\begin{array}{c}\beta \\
(\mathrm{bps} / \mathrm{Hz})\end{array}$ & 12.396 & 34.356 & 13.546 & 37.363 \\
\hline
\end{tabular}




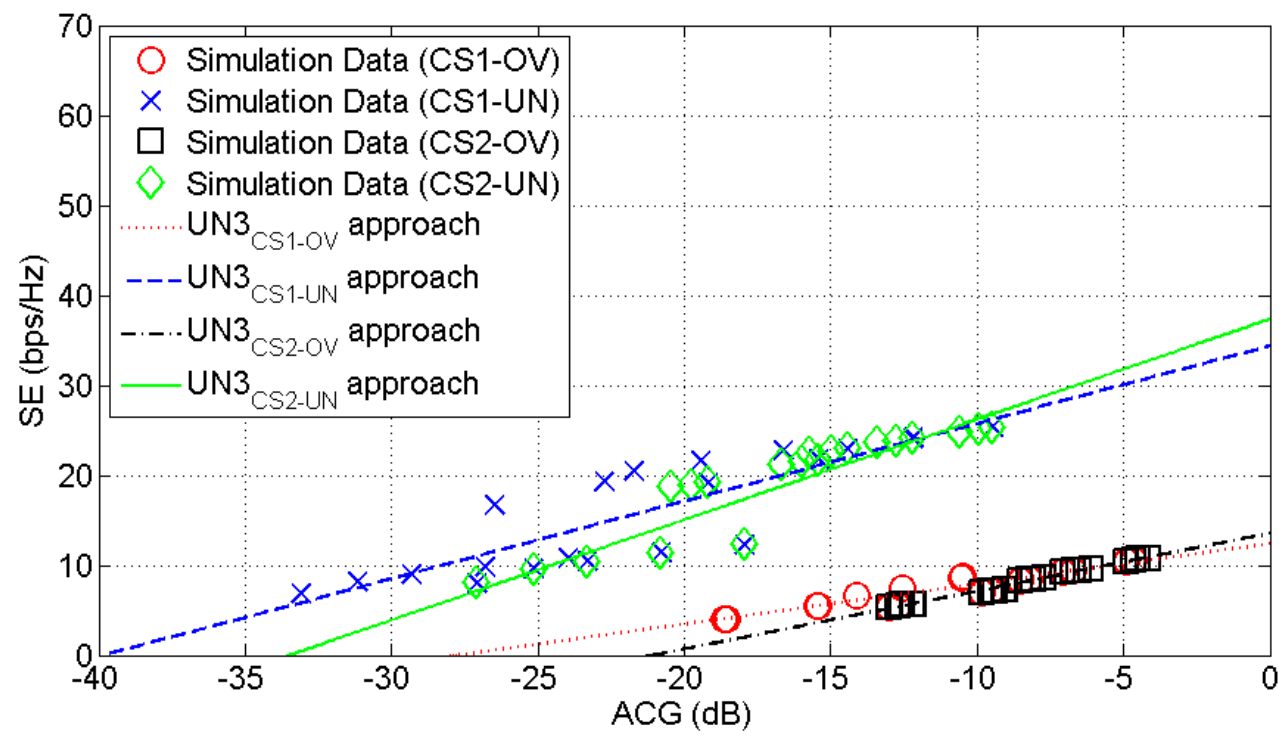

Fig. 5. Scatter plot of SE versus ACG for simulated coupling scheme OV and UN LV and MV BPL channels and various regression approaches and coupling scheme modules.

the BPL topologies of the examined power grid type (i.e., either $\mathrm{OV}$ or $\mathrm{UN}$ ) when $\mathrm{WtG}^{1}, \mathrm{StP}^{1}, \mathrm{WtW}^{2-3}, \mathrm{PtP}^{2-3}$ and $\mathrm{MtM}_{0.7_{-}-0.1_{-}-0.2}^{1-2-2}$ of the applied coupling scheme module (i.e., either CS2 or CS1 module) are used in accordance with Tables 11 and 12.

In Fig. 5, except for the simulation data of Tables 11 and 12, the set of regression trend curves of Table 15, say UN3 ${ }_{\mathrm{CS} 1-\mathrm{OV}}, \mathrm{UN} 3 \mathrm{CS}_{\mathrm{C} 1-\mathrm{UN}}, \mathrm{UN} 33_{\mathrm{CS} 2-\mathrm{OV}}$ and UN3 CS2-UN approaches, are illustrated.

From Fig. 5, several interesting observations concerning the correlation between SE and ACG can be made:

- For the same ACG value, UN distribution BPL networks present higher SE in comparison with OV distribution BPL networks. This is due to the fact that UN environment is a more protected environment concerning: (i) its transmitted EMI to the other radioservices that operate at the same frequency operation band; and (ii) the EMI that receives from the aforementioned radioservices. Therefore, higher IPSD limits are applied in UN distribution BPL networks due to the previous first reason while lower noise PSD is presented in UN distribution BPL networks due to the previous second reason. The favourable operation of UN distribution BPL networks is reflected on the significant $\beta$ difference between UN and OV distribution BPL networks (see also Table 15) for given coupling scheme module.

- Coupling schemes of type 1 produce pairs of SE / ACG values that coincide when CS1 and CS2 module are applied. When coupling schemes of type 2 are examined, CS2 module has as an output SE / ACG points that are located at more upper right positions in comparison with the respective ones of CS1 module when a certain distribution BPL topology is examined. This has as an effect higher inclinations 
of $\mathrm{UN} 3_{\mathrm{CS} 2-\mathrm{OV}}$ and $\mathrm{UN} 3_{\mathrm{CS} 2-\mathrm{UN}}$ curves in comparison with $\mathrm{UN} 3_{\mathrm{CS} 1-\mathrm{OV}}$ and $\mathrm{UN} 3_{\mathrm{CS} 1-}$ uN ones, respectively (see also $\alpha$ of Table 15).

- Despite the differences between UN3 3 CS2-Ov and UN3 3 CS1-Ov curves, whichever of these two curves can offer an approximate estimation of SE and, thus, capacity when the ACG of an overhead distribution BPL topology is available. Similarly to OV distribution BPL networks, UN3 $3_{\mathrm{CS} 2-\mathrm{UN}}$ and $\mathrm{UN} 3_{\mathrm{CS} 1-\mathrm{UN}}$ curves can facilitate the approximate SE estimation when ACG of a UN distribution BPL topology is given.

\subsection{UN1, UN2 and UN3 Approaches - The Impact of CS2 Module}

UN1 and UN2 approaches have been computed either for MIMO or for coupling scheme channels of the OV and UN LV and MV BPL topologies. Especially, in the case of coupling scheme channels, the two different available coupling scheme modules (i.e., CS1 and CS2 module) have also been applied and assessed.

As UN1 approach is concerned, UN1 $1_{\mathrm{MIMO}}, \mathrm{UN} 1_{\mathrm{CS} 1}$ and $\mathrm{UN} 1_{\mathrm{CS} 2}$ approaches differ from other well validated RMS-DS / ACG approaches of the BPL literature since these approaches are exclusively computed with respect to suitable simulation data of

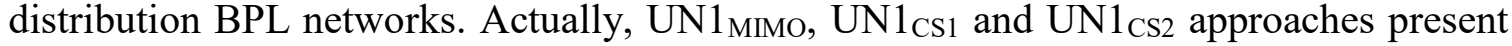
small differences among them due to the different nature of the examined simulation data; UN1 1 MIMO approach comes from the regression analysis of MIMO channel simulation data while $\mathrm{UN} 1_{\mathrm{CS} 1}$ and $\mathrm{UN1} 1_{\mathrm{CS} 2}$ modules also take into consideration the impact of the coupling scheme module and coupling scheme channels. Finally, the impact of CS2 module, which can support more coupling scheme types and more efficient coupling schemes in comparison with CS1 module, is schematically demonstrated through the horizontal curve shift of $\mathrm{UN1} 1_{\mathrm{CS} 1}$ and $\mathrm{UN1} 1_{\mathrm{CS} 2}$ approaches.

As UN2 approach is regarded, UN2 ${ }_{\mathrm{MIMO}}, \mathrm{UN} 2_{\mathrm{CS} 1}$ and $\mathrm{UN} 2_{\mathrm{CS} 2}$ approaches slightly differ each other due to the form of the regression hyperbolic trend curves regardless of the nature of the examined simulated data. Extensively been used in the BPL literature, the regression hyperbolic trend curves seem to better describe MIMO and coupling scheme channels of urban and suburban distribution BPL topologies rather than corresponding channels of "LOS" and rural distribution BPL topologies.

On the basis of the newly proposed UN3 approach, an approximate SE and capacity estimation can be available if an estimate of the average ACG of a distribution BPL network is assumed. Furthermore, this approximation of SE and capacity can be offered with adequate accuracy for OV distribution BPL networks even if the applied coupling scheme is not known; this is due to the fact that $\mathrm{UN} 3{ }_{\mathrm{CS} 2-\mathrm{OV}}$ and $\mathrm{UN} 3_{\mathrm{CS} 1-\mathrm{OV}}$ curves give relatively close results. The same observations can be made for UN distribution BPL networks.

Synoptically, taking under consideration the relatively small differences among $\mathrm{UN} 1, \mathrm{UN} 2$ and UN3 approaches and their universal consideration of the BPL character as described in [17], UN1 ${ }_{\mathrm{CS} 2}, \mathrm{UN} 2_{\mathrm{CS} 2}, \mathrm{UN} 3_{\mathrm{CS} 2-\mathrm{OV}}$ and $\mathrm{UN} 3_{\mathrm{CS} 2-\mathrm{UN}}$ approaches can be further promoted as the basis towards the common statistical handling of: (i) OV and UN LV and MV BPL topologies; (ii) different MIMO BPL channels; (iii) different BPL channels supported by CS1 and CS2 scheme modules; and (iv) different BPL channels of various coupling schemes. 


\section{Conclusions}

This paper has focused on the assessment of the application of CS2 module in $\mathrm{OV}$ and UN LV and MV BPL topologies in terms of four well-known broadband performance metrics (i.e., ACG, RMS-DS, $\mathrm{CB}$ and SE). The results of the aforementioned broadband performance metrics when CS2 modules is applied have been compared against respective ones of CS1 module.

The investigation of the statistical metric comparison results reveal that CS2 module offers significant advantages in comparison with the vintage CS1 module. Indeed, CS2 module supports a plethora of new adaptive coupling schemes (i.e., coupling schemes of the coupling scheme type 3) whose performance can be adjusted in accordance with the needs for higher broadband performance and EMC. Also, CS2 module offers better ACGs for the coupling schemes of the coupling scheme type 2 in comparison with CS1 module. ACG improvement of the coupling schemes of the coupling scheme type 2 is reflected on respective capacity and SE results. Since WtW / PtP coupling scheme channels become almost capacity equivalent to WtG / StP coupling scheme channels, the already-known better EMC performance of WtW / PtP coupling scheme channels against WtG / StP ones can influence the BPL system architecture design concerning the selection of suitable coupling schemes.

As the UN1, UN2 and UN3 approaches of CS2 module are concerned, the fundamental properties of the negative correlation between RMS-DS / ACG, the hyperbolic correlation between RMS-DS / CB and the positive correlation between SE / ACG have been validated in OV and UN LV and MV BPL coupling scheme channels. It has been proven that $\mathrm{UN} 1_{\mathrm{CS} 2}, \mathrm{UN} 1_{\mathrm{CS} 1}$ and $\mathrm{UN} 1_{\mathrm{MIMO}}$ approach curves have small and affordable differences among them, thus allowing the consideration of UN1 ${ }_{\mathrm{CS} 2}$ approach curves as a benchmark curve for the OV and UN LV and MV BPL channels regardless of their type (i.e., MIMO channels, CS1 module coupling scheme channels or CS2 module coupling scheme channels). For the UN2 approach curves, the differences among $\mathrm{UN} 22_{\mathrm{CS} 2}, \mathrm{UN} 2_{\mathrm{CS} 1}$ and $\mathrm{UN} 2_{\mathrm{MIMO}}$ approach curves are even smaller than UN1 approach curves difference due to the mathematical form of the approach. Again, UN2 CS2 approach curves can be applied to efficiently describe coupling scheme and MIMO channels of $\mathrm{OV}$ and UN distribution BPL networks. Finally, for the $\mathrm{UN} 3$ approach curves, it has been proven that $\mathrm{UN} 3 \mathrm{CS2}-\mathrm{OV}$ and $\mathrm{UN} 3 \mathrm{CS1}$-Ov present small differences between them thus permitting the consideration of $\mathrm{UN} 3 \mathrm{CS2}-\mathrm{OV}$ approach curves as an estimation tool for the SE and capacity for the OV and UN LV and MV BPL channels regardless of their type (i.e., CS1 module coupling scheme channels or CS2 module coupling scheme channels). The same assumption is valid in $\mathrm{UN}$ distribution BPL networks when $\mathrm{UN} 3_{\mathrm{CS} 2-\mathrm{UN}}$ approach curve is applied.

\section{CONFLICTS OF INTEREST}

The author declares that there is no conflict of interests regarding the publication of this paper. 


\section{References}

[1] T. A. Papadopoulos, A. I. Chrysochos, E. O. Kontis, R. N. Papadopoulos, and G. K. Papagiannis, "Measurement-based hybrid approach for ringdown analysis of power systems," IEEE Transactions on Power Systems, vol. 31, no. 6, pp. 44354446, 2016.

[2] A. G. Lazaropoulos, "Review and Progress towards the Capacity Boost of Overhead and Underground Medium-Voltage and Low-Voltage Broadband over Power Lines Networks: Cooperative Communications through Two- and Three-Hop Repeater Systems," ISRN Electronics, vol. 2013, Article ID 472190, pp. 1-19, 2013.

[3] A. G. Lazaropoulos, "Review and Progress towards the Common Broadband Management of High-Voltage Transmission Grids: Model Expansion and Comparative Modal Analysis," ISRN Electronics, vol. 2012, Article ID 935286, pp. 1-18, 2012.

[4] S. Galli, A. Scaglione, and Z. Wang, "For the grid and through the grid: the role of power line communications in the smart grid," in Proc. IEEE, vol. 99, no. 6, pp. 998-1027, Jun. 2011.

[5] A. N. Milioudis, G. T. Andreou, and D. P. Labridis, "Detection and Location of High Impedance Faults in Multiconductor Overhead Distribution Lines Using Power Line Communication Devices," IEEE Trans. Smart Grid., vol. 6, no. 2, pp. 894-902, Mar. 2015.

[6] A. G. Lazaropoulos, "Towards Modal Integration of Overhead and Underground Low-Voltage and Medium-Voltage Power Line Communication Channels in the Smart Grid Landscape: Model Expansion, Broadband Signal Transmission Characteristics, and Statistical Performance Metrics (Invited Paper)," ISRN Signal Processing, vol. 2012, Article ID 121628, pp. 1-17, 2012. . [Online]. Available: http://downloads.hindawi.com/journals/isrn/2012/121628.pdf

[7] A. G. Lazaropoulos, "Deployment Concepts for Overhead High Voltage Broadband over Power Lines Connections with Two-Hop Repeater System: Capacity Countermeasures against Aggravated Topologies and High Noise Environments," Progress in Electromagnetics Research B, vol. 44, pp. 283-307, 2012.

[8] C. Cano, A. Pittolo, D. Malone, L. Lampe, A. M. Tonello, and A. G., Dabak, "State of the art in power line communications: From the applications to the medium," IEEE Journal on Selected Areas in Communications, vol. 34, no. 7, pp. 1935-1952, 2016.

[9] M. S. P. Facina, H. A. Latchman, H. V. Poor, and M. V. Ribeiro, "Cooperative InHome Power Line Communication: Analyses Based on a Measurement Campaign," IEEE Trans. Commun., vol. 64, no. 2, pp. 778-789, Feb. 2016.

[10] G. Prasad, L. Lampe, and S. Shekhar, "In-Band Full Duplex Broadband Power Line Communications," IEEE Trans. Comm., vol. 64, no. 9, pp. 3915-3931, Sep. 2016.

[11] A. G. Lazaropoulos and P. G. Cottis, "Transmission characteristics of overhead medium voltage power line communication channels," IEEE Trans. Power Del., vol. 24, no. 3, pp. 1164-1173, Jul. 2009.

[12] M. Antoniali, F. Versolatto, and A. M. Tonello, "An Experimental Characterization of the PLC Noise at the Source," IEEE Trans. Power Del., vol. 31, no. 3, pp. 1068-1075, Jun. 2016. 
[13] R. Lefort, R. Vauzelle, V. Courtecuisse, N., Idir, and A. M. Poussard, "Influence of the MV/LV Transformer Impedance on the Propagation of the PLC Signal in the Power Grid," IEEE Trans. Power Del., vol. 32, no. 3, pp. 1339-1349, Jun. 2017.

[14] A. G. Lazaropoulos and P. G. Cottis, "Capacity of overhead medium voltage power line communication channels," IEEE Trans. Power Del., vol. 25, no. 2, pp. 723-733, Apr. 2010.

[15] A. G. Lazaropoulos and P. G. Cottis, "Broadband transmission via underground medium-voltage power lines-Part I: transmission characteristics," IEEE Trans. Power Del., vol. 25, no. 4, pp. 2414-2424, Oct. 2010.

[16] A. G. Lazaropoulos and P. G. Cottis, "Broadband transmission via underground medium-voltage power lines_-Part II: capacity," IEEE Trans. Power Del., vol. 25, no. 4, pp. 2425-2434, Oct. 2010.

[17] A. G. Lazaropoulos, "Broadband over Power Lines Systems Convergence: Multiple-Input Multiple-Output Communications Analysis of Overhead and Underground Low-Voltage and Medium-Voltage BPL Networks (Invited Paper)," ISRN Power Engineering, vol. 2013, Article ID 517940, pp. 1-30, 2013. [Online]. Available: http://www.hindawi.com/isrn/power.engineering/2013/517940/

[18] T. A. Papadopoulos, C. G. Kaloudas, A. I. Chrysochos, and G. K. Papagiannis, "Application of Narrowband Power-Line Communication in Medium-Voltage Smart Distribution Grids," IEEE Trans. Power Del., vol. 28, no. 2, pp. 981-988, Apr. 2013.

[19] N. Pavlidou, A. J. Han Vinck, J. Yazdani, and B. Honary, "Power line communications: state of the art and future trends," IEEE Commun. Mag., vol. 41, no. 4, pp. 34-40, Apr. 2003.

[20] M. Gebhardt, F. Weinmann, and K. Dostert, "Physical and regulatory constraints for communication over the power supply grid," IEEE Commun. Mag., vol. 41, no. 5, pp. 84-90, May 2003.

[21] A. G. Lazaropoulos, "Towards Broadband over Power Lines Systems Integration: Transmission Characteristics of Underground Low-Voltage Distribution Power Lines," Progress in Electromagnetics Research B, vol. 39, pp. 89-114, 2012.

[22] G. Jee, C. Edison, R. Das Rao, and Y. Cern, "Demonstration of the technical viability of PLC systems on medium- and low-voltage lines in the United States," IEEE Commun. Mag., vol. 41, no. 5, pp. 108-112, May 2003.

[23] A. G. Lazaropoulos, "Broadband transmission and statistical performance properties of overhead high-voltage transmission networks," Hindawi Journal of Computer Networks and Commun., 2012, article ID 875632, 2012.

[24] A. G. Lazaropoulos, "Green Overhead and Underground Multiple-Input MultipleOutput Medium Voltage Broadband over Power Lines Networks: EnergyEfficient Power Control," Springer Journal of Global Optimization, vol. 2012 / Print ISSN 0925-5001, pp. 1-28, Oct. 2012.

[25] A. G. Lazaropoulos, "Overhead and Underground MIMO Low Voltage Broadband over Power Lines Networks and EMI Regulations: Towards Greener Capacity Performances," Elsevier Computers and Electrical Engineering, in press. [Online]. Available: http://www.sciencedirect.com/science/article/pii/S0045790613000293

[26] A. G. Lazaropoulos, "Capacity Performance of Overhead Transmission MultipleInput Multiple-Output Broadband over Power Lines Networks: The Insidious Effect of Noise and the Role of Noise Models (Invited Paper)," 
Trends in Renewable Energy, vol. 2, no. 2, pp. 61-82, Jan. 2016. [Online]. Available: http://futureenergysp.com/index.php/tre/article/view/23

[27] A. G. Lazaropoulos, "Power Systems Stability through Piecewise Monotonic Data Approximations - Part 1: Comparative Benchmarking of L1PMA, L2WPMA and L2CXCV in Overhead Medium-Voltage Broadband over Power Lines Networks," Trends in Renewable Energy, vol. 3, no. 1, pp. 2 - 32, Jan. 2017. [Online]. Available: http://futureenergysp.com/index.php/tre/article/view/29/34

[28] A. G. Lazaropoulos, "Power Systems Stability through Piecewise Monotonic Data Approximations - Part 2: Adaptive Number of Monotonic Sections and Performance of L1PMA, L2WPMA and L2CXCV in Overhead Medium-Voltage Broadband over Power Lines Networks," Trends in Renewable Energy, vol. 3, no. 1, pp. $33-60, \quad$ Jan. 2017. [Online]. Available: http://futureenergysp.com/index.php/tre/article/view/30/35

[29] A. G. Lazaropoulos, "Improvement of Power Systems Stability by Applying Topology Identification Methodology (TIM) and Fault and Instability Identification Methodology (FIIM) - Study of the Overhead Medium-Voltage Broadband over Power Lines (OV MV BPL) Networks Case," Trends in Renewable Energy, vol. 3, no. 2, pp. 102 - 128, Apr. 2017. [Online]. Available: http://futureenergysp.com/index.php/tre/article/view/34/pdf

[30] A. G. Lazaropoulos, "Measurement Differences, Faults and Instabilities in Intelligent Energy Systems - Part 1: Identification of Overhead High-Voltage Broadband over Power Lines Network Topologies by Applying Topology Identification Methodology (TIM)," Trends in Renewable Energy, vol. 2, no. 3, pp. $85-112$, Oct. 2016.

[31] A. G. Lazaropoulos, "Wireless Sensor Network Design for Transmission Line Monitoring, Metering and Controlling: Introducing Broadband over PowerLinesenhanced Network Model (BPLeNM)," ISRN Power Engineering, vol. 2014, Article ID 894628, 22 pages, 2014. doi:10.1155/2014/894628. [Online]. Available: http://www.hindawi.com/journals/isrn.power.engineering/2014/894628/

[32] A. G. Lazaropoulos, "Wireless Sensors and Broadband over PowerLines Networks: The Performance of Broadband over PowerLines-enhanced Network Model (BPLeNM) (Invited Paper)," ICAS Publishing Group Transaction on IoT and Cloud Computing, vol. 2, no. 3, pp. 1-35, 2014. [Online]. Available: http://citeseerx.ist.psu.edu/viewdoc/download;jsessionid=741EE7C15693046FFFF5E9749149F57 9?doi=10.1.1.679.8217\&rep=rep1\&type=pdf

[33] A. G. Lazaropoulos, "New Coupling Schemes for Distribution Broadband over Power Lines (BPL) Networks," Progress in Electrom. Research B (PIERB), vol. 71, pp. 39-54, 2016.

[34] A. G. Lazaropoulos, "A Panacea to Inherent BPL Technology Deficiencies by Deploying Broadband over Power Lines (BPL) Connections with Multi-Hop Repeater Systems," Bentham Recent Advances in Electrical \& Electronic Engineering, vol. 10, no. 1, pp. 30-46, 2017.

[35] P. Amirshahi and M. Kavehrad, "High-frequency characteristics of overhead multiconductor power lines for broadband communications," IEEE J. Sel. Areas Commun., vol. 24, no. 7, pp. 1292-1303, Jul. 2006.

[36] T. Sartenaer, "Multiuser communications over frequency selective wired channels and applications to the powerline access network" Ph.D. dissertation, Univ. Catholique Louvain, Louvain-la-Neuve, Belgium, Sep. 2004. 
[37] P. Amirshahi, "Broadband access and home networking through powerline networks" Ph.D. dissertation, Pennsylvania State Univ., University Park, PA, May 2006.

[38] OPERA1, D44: Report presenting the architecture of plc system, the electricity network topologies, the operating modes and the equipment over which PLC access system will be installed, IST Integr. Project No 507667, Dec. 2005.

[39] DLC+VIT4IP, D1.2: Overall system architecture design DLC system architecture. FP7 Integrated Project No 247750, Jun. 2010.

[40] OPERA1, D5: Pathloss as a function of frequency, distance and network topology for various LV and MV European powerline networks. IST Integrated Project No 507667, Apr. 2005.

[41] S. Galli, "A novel approach to the statistical modeling of wireline channels," IEEE Trans. Commun., vol. 59, no. 5, pp. 1332-1345, May 2011.

[42] S. Galli, "A simplified model for the indoor power line channel," in Proc. IEEE Int. Symp. Power Line Communications and Its Applications, Dresden, Germany, Mar./Apr. 2009, pp. 13-19.

[43] S. Galli, "A simple two-tap statistical model for the power line channel," in Proc. IEEE Int. Symp. Power Line Communications and Its Applications, Rio de Janeiro, Brazil, Mar. 2010, pp. 13-19.

[44] F. Versolatto and A. M. Tonello, "Analysis of the PLC channel statistics using a bottom-up random simulator," in Proc. IEEE Int. Symp. Power Line Communications and Its Applications, Rio de Janeiro, Brazil, Mar. 2010, pp. 236241.

[45] A. M. Tonello, F. Versolatto, and C. Tornelli, "Analysis of impulsive UWB modulation on a real MV test network," in Proc. IEEE Int. Symp. Power Line Communications and Its Applications, Udine, Italy, Apr. 2011, pp. 18-23.

[46] M. Antoniali, A. M. Tonello, M. Lenardon, and A. Qualizza, "Measurements and analysis of PLC channels in a cruise ship," in Proc. IEEE Int. Symp. Power Line Communications and Its Applications, Udine, Italy, Apr. 2011, pp. 102-107.

[47] A. M. Tonello and F. Versolatto, "Bottom-up statistical PLC channel modelingPart II: inferring the statistics," IEEE Trans. Power Delivery, vol. 25, no. 4, pp. 2356-2363, Oct. 2010.

[48] M. Tlich, A. Zeddam, F. Moulin, and F. Gauthier, "Indoor power-line communications channel characterization up to $100 \mathrm{MHz}-$ Part I: one parameter deterministic model," IEEE Trans. Power Del., vol. 23, no. 3, pp. 1392-1401, July 2008.

[49] M. Tlich, A. Zeddam, F. Moulin, and F. Gauthier, "Indoor power-line communications channel characterization up to $100 \mathrm{MHz}-$ Part II: TimeFrequency Analysis," IEEE Trans. Power Del., vol. 23, no. 3, pp. 1402-1409, July 2008.

[50] A. M. Tonello, A. Pittolo, and M. Girotto, "Power line communications: Understanding the channel for physical layer evolution based on filter bank modulation," IEICE Transactions on Communications, vol. 97, no. 8, pp. 14941503, 2014.

[51] J. A. Cortés, F.J. Cañete, L. Díez, and J. L. G. Moreno, "On the Statistical Properties of Indoor Power Line Channels: Measurements and Models," Proc. Int. Symp. on Power Line Commun. and Its App. (ISPLC), pp.271-276, April 2011. 
[52] M. Tlich, A. Zeddam, A. Moulin, and F. Gauthier, "Indoor PowerLine Communications Channel Characterization up to $100 \mathrm{MHz}$ - Part II: TimeFrequency Analysis," IEEE Trans. on Power Del., vol. 23, no. 3, pp. 1402-1409, Jul. 2008.

[53] F. V. A. Pittolo, M. De Piante and A. Tonello, "In vehicle plc: In-car and in-ship channel characterization," IEEE Vehicular Technology Magazine, Aug. 2015.

Article copyright: (C) 2018 Athanasios G. Lazaropoulos. This is an open access article distributed under the terms of the Creative Commons Attribution 4.0 International License, which permits unrestricted use and distribution provided the original author and source are credited. 